Realização de conjuntos como conjuntos de pontos fixos de $G$-deformações

Antônio José Martins Neto

DISSERTAÇÃO APRESENTADA $\mathrm{AO}$

INSTITUTO DE MATEMÁTICA E ESTATÍSTICA

DA

UNIVERSIDADE DE SÃO PAULO

PARA

OBTENÇÃO DO GRAU

$\mathrm{DE}$

MESTRE EM MATEMÁTICA

Área de Concentração: Topologia Algébrica

Orientadora: Profa. Dra. Lucília Daruiz Borsari

-São Paulo, Agosto de 2002- 


\title{
Realização de conjuntos como conjuntos de pontos fixos de $G$-deformações
}

\author{
Este exemplar corresponde à redação \\ final da dissertação devidamente corrigida e \\ defendida por Antônio José Martins Neto \\ e aprovada pela comissão julgadora.
}

São Paulo, 23 de Agosto de 2002

Banca examinadora:

- Profa. Dra. Lucília Daruiz Borsari (Orientadora) - IME - USP

- Prof. Dr. Daciberg Lima Gonçalves - IME - USP

- Prof. Dr. Ozirides Manzolli Neto - ICMC - USP 


\section{Agradecimentos}

À Profa. Lucília Daruiz Borsari pela orientação cordial, precisa, indispensável e paciente.

Ao Prof. Daciberg Lima Gonçalves pela ajuda inestimável e sempre cordial. À minha família.

Às amigas de sempre: Simone e Nadéia.

À Liane e ao Daniel, pela amizade e grata convivência.

$\mathrm{E}$ a todos os que, direta ou indirentamente, contribuíram para a elaboração deste trabalho. 


\title{
Resumo
}

Nesse trabalho apresentamos um resultado de realização de conjuntos como conjuntos de pontos fixos de $G$-deformações.

Demonstramos:(i) Se $G$ é um grupo finito e $M$ é uma $G$-variedade de dimensão finita, compacta e conexa, então dado subconjunto $P \subset \operatorname{int} M$, não vazio, compacto, $G$-invariante e com intersecção não vazio com toda componente conexa de $M_{H}$, para cada $H \leq G \operatorname{com} M_{H} \neq \emptyset$, existe uma $G-$ deformação $f: M \rightarrow M$ cujo conjunto de pontos fixos é $P$. (ii) Apresentamos um exemplo, devido a D. Ferrario, de um grupo finito $G$ e uma $G$-variedade $M$, compacta, conexa e de dimensão finita em que as identidades de $M^{H}$, para $H \leq G$, podem ser deformadas a aplicações livres de pontos fixos, no entanto a identidade de $M$ não pode ser $G$-deformada a uma aplicação livre de pontos fixos.

\begin{abstract}
In this work we present a result about realization of sets as fixed point sets of $G$-deformation.

We show:( $i$ ) If $G$ is a finite group and $M$ is a $n$ dimensional $G$-manifold, compact and connected, then given a non empty subset $P \subset \operatorname{int} M$, compact, $G$-invariant such that $P \cap C \neq \emptyset$ for each connected component $M_{H}$ with $M_{H} \neq \emptyset$, there exists a $G$-deformation $f: M \rightarrow M$ such that the fixed point set of $F$ is $P$. (ii) We show a example, due to D. Ferrario, of a finite group $G$ and a finite dimensional $G$-manifold, compact and connected such that the indentity of $M^{H}$, for $H \leq G$, can be deformed to fixed point free map, but the identity of $M$ can't be $G$-deformed to a fixed point free map.
\end{abstract}


O objetivo desse trabalho é a apresentação de um resultado de realização de conjuntos não vazios como conjuntos de pontos fixos de $G$-deformações em $G$-variedades.

Em 1974 Helga Schirmer (cf. [Schirmer]) mostrou, no contexto não equivariante, que todo subconjunto fechado e não vazio de um poliedro $K$ pode ser o conjunto de pontos fixos de uma aplicação de $K$ em si mesmo, desde que o poliedro satisfaça determinada condição de conexidade. Em particular foi mostrado que todo fechado não vazio de uma variedade triangularizável, com ou sem bordo, pode ser realizado como conjunto de pontos fixos de uma aplicação da variedade em si mesma. Shichang Wang e Tong Xue mostraram em 1983 (cf.[Wang]) o mesmo resultado para variedades compactas: dada uma variedade $\mathcal{C}^{\infty} M$, compacta em que todas as componente do bordo tem característica de Euler nula, se a variedade possuir bordo, é possível obter um campo de vetores sobre $M$ com apenas uma singularidade e a partir deste campo obtém-se, via o fluxo do campo, um aplicação $f: M \rightarrow M$ que tem como conjunto de pontos fixos o conjunto previamente dado.

No artigo A note on equivariant vector fields de 1991, Boju Jiang (cf. [Jiang]), apresentou uma generalização do resultado de realização de [Wang] para o contexto equivariante, para tanto, utilizando e generalizando alguns resultados apresentados em [Halpern]. Os resultados em teoria de pontos fixos obtidos em [Jiang] se dá mediante a construção de campos de vetores equivariantes que tem como singularidades o conjunto a ser realizado como conjunto de pontos fixos e além disso não possui órbitas não singulares fechadas, e portanto, não possui pontos periódicos. Tomando-se o semi-fluxo 
do campo obtemos a aplicação desejada.

Um problema relacionado ao de realização de conjuntos como conjuntos de pontos fixos é o da obtenção de uma aplicação sem pontos fixos na classe de homotopia de uma dada aplicação. Um resultado clássico garante que uma variedade compacta de classe $\mathcal{C}^{\infty}$ admite um homeomorfismo (difeomorfismo) sem pontos fixos se, e somente se, a caracteristica de Euler da variedade é zero. Pode-se, ainda, garantir que o homeomorfismo obtido esteja na classe de homotopia da identidade. Em [BG], [FW], [Vidal] e [Wilczyński] o problema da deformação de uma aplicação a uma aplicação livre de pontos fixos foi estudado no contexto equivariante. Em [Jiang] o problema acima mencionado é, também, estudado no caso da identidade. A motivação original de [Jiang] foi a remoção de várias hipóteses assumidas nos trabalhos de $[\mathbf{B G}],[\mathbf{F W}]$, [Vidal] e [Wilczyński]. Porém vários resultados obtidos mostraram-se não corretos: Davide Ferrario (cf. [Ferrario]) construiu um exemplo de $G$-variedade em que a identidade não pode ser $G$-deformada a uma aplicação livre de pontos fixos, apesar da variedade satisfazer todas as hipóteses pedidas em [Jiang]. Para a análise de tal exemplo não empregaremos as técnicas originais de seu autor, pois fogem completamente do contexto dessa dissertação; empregaremos um critério dado por Komiya em [Komiya] que tem uma relação maior com a linha dessa dissertação.

Este trabalho está organizado do seguinte modo:

No capítulo I apresentamos as várias noções e resultados que serão necessários para a demonstração do resultado principal. No capítulo II provamos os seguintes resultados: 
Teorema Sejam $M$ uma $G$-variedade com ou sem bordo e $P \subset$ intM um subconjunto compacto, não vazio e G-invariante. Suponhamos que, para todo subgrupo $H$ de $G$, o subconjunto $P$ tenha intersecção não vazia com todas as componentes de pontos fixos de isotropia $H$, se $M_{H} \neq \emptyset$. Então existe um $G$-campo de vetores $V$ em $M$ tal que $Z_{V}=P$ e cujas órbitas (do campo de vetores) não são fechadas.

Corolário (Realização de conjuntos como conjuntos de pontos fixos de uma $G$-deformação) Sejam $M$ uma $G$-variedade com ou sem bordo e $P \subset$ intM um subconjunto compacto e $G$-invariante com interseç̧ão não vazia com toda componente não vazia de pontos fixos de isotropia $H$, para todo subgrupo $H$ de $G$. Então $P$ é o conjunto de pontos fixos de uma G-deformação.

O capítulo III é dedicado à apresentação do exemplo de D. Ferrario e sua análise utilizando o resultado de [Komiya] 


\section{Sumário}

1 Preliminares $\quad 9$

1.1 Ações de Grupos . . . . . . . . . . . . . . . . . . . . 9

1.2 Grupos de Transformações . . . . . . . . . . . . . . . . . 11

1.3 Pontos Fixos . . . . . . . . . . . . . . . . . . 13

1.4 Espaço de Órbitas . . . . . . . . . . . . . . . . . . . 13

$1.5 G$-Variedades ......................... 14

1.6 G-Funções de Morse . . . . . . . . . . . . . . . . . . . . 21

2 Realização de Conjuntos de Pontos Fixos 23

2.1 Lema da Absorção . . . . . . . . . . . . . . . . . . . . . 23

2.2 Retração Equivariante em $D^{m} \ldots$. . . . . . . . . . . . 29

2.3 Realização de Conjuntos como Conjuntos de Pontos Fixos de uma $G$-deformação . . . . . . . . . . . . . . 40

3 O Exemplo de D. Ferrario $\quad 44$ 


\section{Capítulo 1}

\section{Preliminares}

\subsection{Ações de Grupos}

Definimos, nessa seção, o conceito de ação de um grupo num conjunto e damos suas principais propriedades. Para as definições e proposições em que não há uma referência específica damos como referência [Bredon], [Kawakubo] e [tD].

DEFINIÇÃO 1.1.1. Sejam $G$ um grupo e $X$ um conjunto. Uma ação de $G$ em $X$ é uma função $\varphi: G \times X \rightarrow X$ satisfazendo as duas condições abaixo:

(1) $\varphi(e, x)=x$, para todo $x \in X$, sendo e a identidade do grupo $G$;

(2) $\varphi\left(g_{1}, \varphi\left(g_{2}, x\right)\right)=\varphi\left(g_{1} g_{2}, x\right)$, para todos $g_{1}, g_{2}$ em $G$ e todo $x \in X$.

Nesse caso dizemos que $X$ é um $G$-conjunto com ação $\varphi$.

Por simplicidade de notação, é usual escrever $g x$ para indicar $\varphi(x)$. Usando esta notação as condições (1) e (2) acima se escrevem como:

(1) $e x=x$ para todo $x \in X$, sendo $e$ a identidade do grupo $G$;

(2) $g_{1}\left(g_{2} x\right)=\left(g_{1} g_{2}\right) x$ para todo $g_{1}, g_{2} \in G$ e todo $x \in X$.

Para cada $g$ em $G$ a aplicação $L_{g}: X \rightarrow X$ dada por $L_{g}(x)=g x$, chamada de translação à esquerda por $g$ é uma aplicação bijetora cuja inversa é a aplicação $L_{g^{-1}}$. 
DEFINIÇÃO 1.1.2. (1) Para cada ponto $x \in X$ o subconjunto $G(x)$ dado por $G(x):=\{g x \mid g \in G\}$ é chamado de órbita do ponto $x$. Outra notação usada é Gx.

(2) Dado $x \in X$, o conjunto $G_{x}:=\{g \in G \mid g x=x\}$ é chamado de grupo de isotropia de $x$.

(3) Dizemos que o ponto $x \in X$ é um ponto fixo da ação de $G$ em $X$ se $g x=x$, para todo $g \in G$. O conjunto de todos os pontos fixos da ação de $G$ será denotado por $X^{G}$.

Abaixo estão listadas algumas propriedades básicas dos cojuntos acima definidos:

PROPOSIÇÃO 1.1.3. O grupo de isotropia $G_{x}$ é um subrupo de $G$.

PROPOSIÇÃO 1.1.4. Dados $x, y \in X$, temos $G(x)=G(y)$ ou $G(x) \cap G(y)=\emptyset$. E, portanto, a relação $x \sim y \Longleftrightarrow y \in G(x)$ é uma relação de equivalência sobre $X$ cujas classes de equivalência são as órbitas da ação de $G$ em $X$.

PROPOSIÇÃO 1.1.5. Dados $g \in G$ e $x \in X$ temos $G_{g x}=g G_{x} g^{-1}$.

A proposição acima significa que o grupo de isotropia dos pontos de uma mesma órbita são conjugados e que todos os conjugados são realizados como subgrupos de isotropia.

DEFINIÇÃO 1.1.6. Sejam $X$ e $Y$ dois G-conjuntos com ações $\varphi$ e $\psi$ respectivamente. Uma aplicação $f: X \rightarrow Y$ é chamada de G-equivariante, ou uma $G$-aplicação se comutar com ação do grupo, isto é, se para todo $g \in G$ e todo $x \in X$ tivermos $f(\varphi(g, x))=\psi(g, f(x))$, ou simplesmente $f(g x)=g f(x)$ subtendendo-se as ações $\varphi$ e $\psi$. Uma função $f: X \rightarrow \mathbb{R} e ́$ dita invariante se para todo $x \in X$ e todo $g \in G$ tivermos $f(g x)=f(x)$, isto 
é, uma função invariante é uma função equivariante do $G$-conjunto $X$ na reta real, considerando nesta a ação trivial de G. Um G-isomorfismo é uma G-aplicação bijetora.

DEFINIÇÃO 1.1.7. Dados $g \in G$ e $Y \subseteq X$, definimos $g Y:=\{g y \mid y \in Y\}$ $e G Y:=\{g y \mid g \in G$ e $y \in Y\}$. Dizemos que $Y \subseteq X$ é $G$-invariante se para todo $g \in G$ ocorrer $g Y \subseteq Y$, ou de forma equivalente, se $G Y=Y$.

PROPOSIÇÃO 1.1.8. Se $Y \subseteq X$ é subconjunto $G$-invariante, então $G$ induz em $Y$ uma $G$-ação. Em particular, $G$ induz em cada órbita uma $G$ ação.

DEFINIÇÃO 1.1.9. Uma ação de $G$ em $X$ é:

(1) trivial se $G_{x}=G$ para todo $x \in X$.

(2) livre se $G_{x}=\{1\}$ para todo $x \in X$.

(3) semi-livre se $G_{x}=G$ ou $G_{x}=\{1\}$, para todo $x \in X$.

(4) transitiva se exitir apenas uma órbita.

(5) efetiva se $\bigcap_{x \in X} G_{x}=\{1\}$

DEFINIÇÃO 1.1.10. Sejam $H$ e $K$ subgrupos de um grupo $G$. Dizemos que $H$ e $K$ são conjugados em $G$, ou simplesmente conjugados, se existir um elemento $g \in G$ tal que $H=g K^{-1}$. Nesse caso escrevemos $H \sim K$. A relação de conjugação é uma relação de equivalência no conjunto dos subgrupos de $G$, cuja classe de equivalência de um subgrupo $H$ é denotada por $(H)$.

\subsection{Grupos de Transformações}

Estaremos interessados em ações $\mathcal{C}^{\infty}$ em variedades diferenciáveis. Para definirmos tal conceito é necessário que o grupo possua uma estrutura topológica compatível com sua estrutura algébrica. Nesta seção introduzimos os grupos topológicos e enunciamos as propriedades necessárias para uso posterior. 
DEFINIÇÃO 1.2.1. Um grupo $G$ é dito um grupo topológico se é espaço topológico Hausdorff e as aplicações $m: G \times G \rightarrow G$ e $i: G \rightarrow G$ dadas por $m(x, y)=x y$ e $i(x)=x^{-1}$ são contínuas:

Podemos substituir a necessidade da continuidade das duas aplicações $m$ e $i$ pela continuidade da aplicação $r: G \times G \rightarrow G$ dada por $r(x, y)=x y^{-1}$.

Notamos que todo grupo pode ser tranformado em um grupo topológico, bastando para tanto muní-lo com a topologia discreta. Assumiremos que a topologia considerada num grupo finito é, sempre, a topologia discreta.

Seja $H$ um subgrupo do grupo topológico $G$. Com a topologia induzida de $G$, o subgrupo $H$ transforma-se de modo natural em um grupo topológico. Se como subespaço de $G$, o subgrupo $H$ for fechado, aberto ou discreto, diremos que $H$ é um subgrupo fechado, subgrupo aberto ou um subgrupo discreto, respectivamente.

DEFINIÇÃO 1.2.2. O normalizador do subgrupo $H$ de $G$ é denotado por $N(H)$ ou $N H$ e é definido por $N(H):=\left\{g \in G \mid g^{-1} H g=H\right\}$. O grupo $H$ é normal em $N(H)$ e o grupo quociente $N(H) / H$, denotado por $W H$, é o grupo de Weyl de $H$.

DEFINIÇÃO 1.2.3. Uma ação do grupo topológico $G$ no espaço topológico $X$ é uma ação contína do grupo $G$ no espaço $X$. Nesse caso dizemos que $X$ é um $G$-espaço. Se $\varphi$ é a ação considerada, então a tripla $(X, G, \varphi)$ é chamada de grupo de transformações.

Com este novo requisito, as aplicações e subconjuntos anteriormente definidos ganham propriedades extras.

PROPOSIÇÃO 1.2.4. Seja $X$ um $G$-espaço com ação $\varphi: G \times X \rightarrow X$. Para cada $g \in G$ a translação $x \in X \stackrel{L_{g}}{\longmapsto} g x \in X$ é um homeomorfismo de $X$. 
PROPOSIÇÃO 1.2.5. Se X é um espaço topológico $T_{1}$, então o grupo de isotropia $G_{x}$ é fechado, qualquer que seja $x \in X$.

DEFINIÇÃO 1.2.6. Sejam $D$ um subconjunto do grupo topológico $G$ e $Y$ um subconjunto do $G$-espaço $X$. Definimos $D Y:=\{d y \mid d \in D$ e $y \in Y\}$.

PROPOSIÇÃO 1.2.7. Seja $X$ um $G$-espaço com ação $\varphi$. Então a aplicação $\varphi$ é aberta. Se G é compacto, então $\varphi$ é fechada.

\subsection{Pontos Fixos}

Dada uma ação de um grupo $G$ num espaço topológico $X$ e dado um subgrupo $H$ de $G$ definimos: $X^{H}:=\left\{x \in X \mid H \subseteq G_{x}\right\}=\{x \in X \mid h x=x, \forall h \in H\}$ e $X_{H}:=\left\{x \in X \mid G_{x}=H\right\}$. O conjunto $X^{H}$ é o conjunto de pontos fixos de $H$ e $X_{H}$ é o conjunto dos pontos de isotropia $H$.

PROPOSIÇÃO 1.3.1. Seja $(X, G, \varphi)$ um grupo de transformações. Se $X$ é Hausdorff, então $X^{H}$ é fechado qualquer que seja o subgrupo $H$ de $G$.

Sejam $(X, G, \varphi)$ grupo de transformções topológicas e $H<G$. De modo geral $G$ não age em $X^{H}$, devido ao fato de $X^{H}$ não ser, necessariamente, $G$-invariante. Um dos modos de impor a invariância de $X^{H}$ é tomar $H \triangleleft G$. Temos a proposição seguinte:

PROPOSIÇÃO 1.3.2. $X^{H}$ é $N(H)$-invariante. Portanto se $H \triangleleft G$, então $X^{H}$ é G-invariante.

\subsection{Espaço de Órbitas}

Dados um grupo de transformações $(X, G, \varphi)$ e um ponto $x \in X$, a órbita de $x$ é $G$-isomorfa ao quociente $G / G_{x}$. É importante saber quando esse 
$G$-isomorfismo é um $G$-homeomorfismo. Tomando $G / G_{x}$ com a topologia quociente vale o resultado abaixo.

PROPOSIÇÃO 1.4.1. Se $G$ é compacto e $X$ é Hausdorff, então $G / G_{x}$ e $G(x)$ são $G$-homeomorfos.

DEFINIÇÃO 1.4.2. O espaço de órbitas de uma G-ação num espaço topológico $X$ é o espaço quociente $X / G$ dado pela relação de equivalência $x \sim y \Longleftrightarrow \exists g \in G$ com $y=g x$.

PROPOSIÇÃ̃O 1.4.3. A projeção $\pi: X \rightarrow X / G$ é uma aplicação aberta.

A proposição seguinte estabelece uma série de propriedades do espaço de órbitas quando $G$ é compacto e $X$ é Hausdorff, compacto ou localmente compacto.

PROPOSIÇÃO 1.4.4. Seja $G$ um grupo topológico compacto e X um G-espaço. Então valem:

(1) A projeção $\pi: X \rightarrow X / G$ é uma aplicação fechada.

(2) Se $X$ é Hausdorff, então X/G também é Hausdorff.

(3) $\pi: X \rightarrow X / G$ é própria (a imagem inversa de compacto é compacto).

(4) $X$ é localmente compacto $\Longleftrightarrow X / G$ é localmente compacto.

\section{$1.5 G$-Variedades}

Adotaremos, em tudo o que segue, o termo variedade para designar variedade diferenciável de classe $\mathcal{C}^{\infty}$ sem bordo. Nas ocasiões em que estivermos considerando uma variedade com bordo indicaremos tal fato explicitamente.

DEFINIÇÃO 1.5.1. Um grupo de Lie é um grupo topológico munido de uma estrutura de variedade diferenciável $\mathcal{C}^{\infty}$ de modo que as operações do grupo são de classe $\mathcal{C}^{\infty}$. 
Observamos que um grupo finito com a topologia discreta é um grupo de Lie.

DEFINIÇÃO 1.5.2. Sejam G um grupo de Lie e $M$ uma variedade com ou sem bordo de classe $\mathcal{C}^{\infty}$. Uma ação $\mathcal{C}^{\infty}$ do grupo $G$ na variedade $M$ é uma aplicação $\phi: G \times M \rightarrow M$ de classe $\mathcal{C}^{\infty}$ que também é uma ação de $G$ em $M$. Nesse caso diremos que $G$ age diferencialvelmente em $M$ e que $M$ é uma G-variedade.

Em tudo o que segue suporemos que as variedades, ações e aplicações consideradas são de classe $\mathcal{C}^{\infty}$.

PROPOSIÇÃ̃o 1.5.3. Seja $\varphi: G \times M \rightarrow M$ uma ação de $G$ em $M$. Então, para todo $g \in G$, as translações à esquerda $x \in M \stackrel{L_{g}}{\longmapsto} g x \in M$ são $G-$ difeomorfismos.

A proposição seguinte é extremamente útil pois caracteriza os conjuntos de pontos fixos pelos subgrupos de isotropia de uma ação.

PROPOSIÇÃ̃ 1.5.4. Sejam $M$ uma G-variedade com ou sem bordo e $G$ um. grupo de Lie compacto. Para cada subgrupo $H$ de $G$ os conjuntos $M_{H} e$ $M^{H}$ são subvariedades de $M$.

DEFINIÇÃO 1.5.5. Sejam $f_{1}$ e $f_{2}$ duas $G$-aplicaçốes da G-variedade $M$ na G-variedade $N$. Dizemos que $f_{1}$ e $f_{2}$ são G-homotópicas se existir homotopia $H: M \times I \rightarrow N$ entre $f_{1}$ e $f_{2}$ tal que a aplicação $H_{t}: M \rightarrow M$ dada por $H_{t}(x)=H(x, t)$ é $G$-equivariante para todo $t \in I$.

A seguir apresentamos alguns conceitos básicos de sistemas dinâmicos que serão generalizados, posteriormente, para o caso equivariante. 
DEFINIÇÃO 1.5.6. Sejam $M$ uma variedade e $X$ um campo de vetores em $M$. Uma curva integral do campo $X$, ou simplesmente curva integral, passando por $p \in M$ é uma curva, de classe $\mathcal{C}^{\infty}, \alpha: I \rightarrow M$ tal que $\alpha(0)=p$ e para todo $t \in I$ vale $\alpha^{\prime}(t)=X(\alpha(t))$, sendo $I$ um intervalo aberto com $0 \in I$. Uma curva integral passando por $p$ é dita maximal se seu domínio contiver o domínio de qualquer outra curva integral passando por $p$. Por uma curva integral maximal entendemos uma curva integral maximal que passa por algum ponto $p \in M$.

PROPOSIÇÃO 1.5.7. Seja $X$ um campo numa variedade $M$. Para cada $p \in M$, existe uma única curva integral maximal passando por $p$. (cf. [Palis], pag. 21)

DEFINIÇÃO 1.5.8. Um campo de vetores sobre uma variedade é chamado de completo se todas as suas curvas integrais maximais estiverem definidas em toda a reta $\mathbb{R}$.

DEFINIÇÃO 1.5.9. Um fluxo global sobre uma variedade $M$, com ou sem bordo, é uma aplicação $\phi: \mathbb{R} \times M \rightarrow M$ de classe $\mathcal{C}^{\infty}$ tal que:

1. $\forall x \in M, \phi(0, x)=x e$

2. $\forall t, s \in \mathbb{R}, \forall x \in M, \phi(t+s, x)=\phi(t, \phi(s, x))$

Para cada $x \in M$ a aplicação $\phi_{x}: \mathbb{R} \rightarrow M$ é uma curva $\mathcal{C}^{\infty}$ em $M$ tal que $\phi_{x}(0)=x$ e para todo $t \in \mathbb{R}$ a aplicação $\phi_{t}: M \rightarrow M$ é um difeomorfismo de classe $\mathcal{C}^{\infty}$; em particular $\phi_{0}=i d_{M}$. (cf. [Palis], pag. 24)

PROPOSIÇÃO 1.5.10. Seja X um campo de vetores numa variedade compacta. Então existe um único fluxo global $\phi: \mathbb{R} \times M \rightarrow M$ tal que

$$
\frac{\partial \phi}{\partial t}(s, x)=X(\phi(s, x)), \forall s \in \mathbb{R}, \forall x \in M
$$


Tal fluxo é chamado de fluxo determinado pelo campo X. Em particular $X$ é completo.(cf. [Palis], pag. 22)

DEFINIÇÃO 1.5.11. Seja $M$ uma G-variedade com ou sem bordo. Podemos munir o fibrado tangente de $M, T(M)$, de uma ação de $G$ :

$$
\begin{gathered}
G \times T(M) \rightarrow T(M) \\
\left(g, X_{p}\right) \mapsto g X_{p}:=d\left(L_{g}\right)_{p}\left(X_{p}\right)
\end{gathered}
$$

Sendo $d\left(L_{g}\right)_{p}$ a derivada, no ponto $p \in M$, da translação à esquerda por $g$ que, por comodidade de notação será indicada por $d g_{p}$.

DEFINIÇÃO 1.5.12. Um $G$ campo de vetores numa G-variedade é um campo de vetores $X: M \rightarrow T M$ equivariante, ou seja, para cada $g \in G$, cada $p \in M$ e cada $X_{p} \in T_{p} M$ vale: $X_{g p}=g X_{p}\left(=d g_{p}\left(X_{p}\right)\right)$.

PROPOSIÇÃO 1.5.13. Sejam $M$ uma G-variedade compacta, $X$ um $G$ campo e $\phi$ o fluxo determinado por $X$. Então $\phi$ é G-equivariante, isto é, $\phi(t, g x)=g \phi(t, x), \forall t \in \mathbb{R}, \forall x \in M$.

\section{Prova:}

Seja $\phi: \mathbb{R} \times M \rightarrow M$ o fluxo global determinado pelo campo $X$. Dados $x \in M$ e $g \in G$, consideremos as curvas $\alpha(t):=g \phi_{x}(t)$ e $\beta(t):=\phi_{g x}(t, x), \forall t \in \mathbb{R}$. Então:

(a) $\alpha(0)=g \phi(0, x)=g x=\phi(0, g x)=\phi_{g x}(0)=\beta(0)$

(b) $\alpha^{\prime}(s)=\frac{d\left(g \phi_{x}\right)}{d t}(s)=d g_{\phi_{x}(s)} \frac{d\left(\phi_{x}\right)}{d t}(s)=d g_{\phi_{x}(s)} \frac{\partial \phi}{\partial t}(s, x)$

$=d g_{\phi_{x}(s)} X\left(\phi_{x}(s, t)\right)=X\left(g \phi_{x}(s, t)\right)=X(\alpha(s))$

Mas por outro lado $\beta^{\prime}(s)=\frac{d \phi_{g x}}{d t}=X\left(\phi_{g x}\right)=X(\beta(s))$ 
Pela unicidade das curvas integrais maximais, Proposição 1.5.7, temos $\alpha(t)=$ $\beta(t), \forall t \in \mathbb{R}$. Já que $x \in M$ e $g \in G$ eram arbitrários, segue a equivariância do fluxo.

DEFINIÇÃO 1.5.14. Seja $M$ uma variedade com ou sem bordo. Uma métrica riemanniana em $M$ é um produto interno de classe $\mathcal{C}^{\infty}$ no fibrado tangente $T(M)$ de $M$. Denotaremos a métrica riemanniana escolhida por $<,>$. Portanto se $X_{p}$ e $Y_{p}$ são elementos de $T_{p} M$, denotaremos por $\left\langle X_{p}, Y_{p}\right\rangle_{p}$ o valor de $<,>$ em tais elementos. Se $M$ é uma $G$-variedade e para todo $p \in M$, a métrica riemanniana satisfaz $\left\langle g X_{p}, g Y_{p}\right\rangle_{g p}=\left\langle X_{p}, Y_{p}\right\rangle_{p}$, com $X_{p} e$ $Y_{p}$ em $T_{p} M$, então dizemos que a métrica dada é uma métrica riemanniana $G$-invariante. Uma $G$-variedade riemanniana é uma $G$-variedade equipada com uma métrica riemanniana $G$-invariante.

PROPOSIÇÃO 1.5.15. Sejam $M$ uma G-variedade com ou sem bordo e $G$ grupo de Lie compacto. Então $M$ admite uma métrica riemanniana $G-$ invariante.(cf. [Bredon], pag. 304)

DEFINIÇÃO 1.5.16. Sejam $M$ uma G-variedade riemanniana, com ou sem bordo, $X$ um $G$-campo de vetores e $f: M \rightarrow \mathbb{R}$ uma função invariante. Para cada $p \in M$, existe um único vetor $v_{p} \in T_{p} M$ tal que $\left\langle v_{p}, X_{p}\right\rangle_{p}=$ $d f_{p}\left(X_{p}\right)$, para todo $X_{p} \in T_{p} M$. O campo que associa a cada $p \in M$ o vetor $v_{p}$ é $\mathcal{C}^{\infty}$ e é chamado de campo gradiente, ou simplesmente de gradiente, da função f. Notação $\nabla f$ ou gradf. (cf. [Palis], pag. 27)

DEFINIÇÃO 1.5.17. Sejam $M$ uma $G$-variedade compacta, X um campo de vetores sobre $M$ e $\phi$ o fluxo determinado por $X$. Dado $p \in M$, a órbita de $p$ é o conjunto $\mathcal{O}(p):=\{\varphi(t, p) \mid t \in \mathbb{R}\}$. Se $X_{p}$ é uma singularidade do campo, isto é, $X_{p}=0$, então a órbita de $p$ fica reduzida ao ponto $p$ e dizemos que a órbita é uma órbita singular. Se $X_{p}$ não é uma singularidade 
do campo, a curva $\alpha(t)=\phi_{x}(t)$ é uma imersão de $\mathbb{R}$ em $M$. Se $\alpha$ não é injetora, existe um número real $T>0$ tal que $\alpha(T)=\alpha(0)$ e $\alpha(t) \neq p$ para $0<t<T$. Neste caso a órbita de $p$ é difeomorfa a $S^{1}$ e dizemos que é uma órbita fechada de período T. Se a órbita não for singular nem fechada, diremos que é uma órbita regular. Portanto uma órbita regular é a imagem de uma imersão injetora de $\mathbb{R}$.(cf. [Palis], pag. 24)

PROPOSIÇÃO 1.5.18. Dada uma função $f: M \rightarrow \mathbb{R}$ invariante da $G-$ variedade $M$ em $\mathbb{R}$, o campo de vetores $\nabla f$ é $G$-equivariante.

\section{Prova:}

$\langle g \nabla f, v\rangle_{g p}=\left\langle\nabla f, g^{-1} v\right\rangle_{p}=d f_{p}\left(g^{-1} v\right)=d f_{p} \circ d g_{g p}^{-1}(v)=$ $=d\left(f \circ g^{-1}\right)_{g p}(v)=d f_{g p}(v)=\left\langle\nabla_{g p}, v\right\rangle_{g p}$, para todo $v \in T_{g p} M$.

Logo $g \nabla f_{p}=\nabla f_{g p}$.

PROPOSIÇÃO 1.5.19. Sejam $M$ uma variedade compacta e $f: M \rightarrow \mathbb{R}$ uma função $\mathcal{C}^{\infty}$. As órbitas não singulares do campo $\nabla f$ não são fechadas.

\section{Prova:}

Sejam $\phi: \mathbb{R} \times M \rightarrow M$ o fluxo determinado pelo $\nabla f$ e $p \in M$ tal que $\mathcal{O}(p)$ é não singular. Seja $\alpha$ a curva integral maximal do campo que passa por $p$. Então $(f \circ \alpha)^{\prime}(t)=d f_{q}\left(\alpha^{\prime}(t)\right)=d f_{q}\left(\nabla f_{q}\right)=\left\langle\nabla f_{q}, \nabla f_{q}\right\rangle=\left\|\nabla f_{q}\right\|^{2}$, sendo $q=\alpha(t)$. Portando $f$ é estritamente crescente ao longo da órbita de $p$, em particular a órbita não é fechada.

DEFINIÇÃO 1.5.20. Sejam $M$ uma variedade de dimensão $M$ com bordo, $p \in \partial M$ e $x_{p} \in T_{p} M$. Seja $(U, \varphi)$ uma carta local em torno do ponto $p$, isto é, $\varphi$ é um difeomorfismo de um aberto $U$ de $M$, contendo o ponto $p$, num aberto $V$ de $\mathbb{R}_{+}^{m}:=\left\{\left(x_{1}, \cdots, x_{m-1}, 0\right) \mid x_{1}, \cdots, x_{m-1} \in \mathbb{R}\right\} . A$ expressão de $X_{p} \in$ $T_{p} M$ em termos da carta $(U, \varphi)$ é: $X_{p}=\sum_{i=1}^{m} a_{i} \frac{\partial \varphi}{\partial x_{i}}(p)$, sendo $a_{i}=X_{p}\left(x_{i}\right)$. 
Dizemos que $X_{p}$ aponta para dentro se $a_{m}>0$ e dizemos que $X_{p}$ aponta para fora se $a_{m}<0$. Pode-se mostrar que a definição é independente da escolha da carta local tomada.(cf. [Boothby], pag. 254)

Seja $M$ uma variedade com bordo e $X$ um campo de vetores em $M$. Dependendo do comportamento do campo nos pontos do bordo pode não haver fluxo global para o campo, mais ainda: nos pontos do bordo as curvas integrais maximais podem estar definidas apenas para $t=0$. Uma situação um pouco melhor ocorrerá se o campo apontar para dentro em todos os pontos do bordo e a variedade for compacta. Nesse caso poderemos definir o semi-fluxo global do campo $X$.

DEFINIÇÃO 1.5.21. Seja $M$ uma variedade com bordo. Seja $\widetilde{M}$ uma cópia de $M$. Identificando $M$ e $\widetilde{M}$ através do bordo obtemos uma variedade sem bordo, que será denotada por $2 M$. Se $M$ é compacta também é compacta a variedade $2 M$.

PROPOSIÇÃO 1.5.22. Sejam $M$ uma variedade compacta com bordo e $X$ um campo de vetores em $M$. Mergulhando $M$ em $N=2 M$ podemos estender o campo $X$ a um campo $Y$, de classe $\mathcal{C}^{\infty}$, definida em toda a variedade $N$.(cf. [Hirsch], pag. 151)

Suponha que na proposição acima o campo $X$ aponte para dentro nos pontos do bordo de $M$. Seja $\phi: \mathbb{R} \times 2 M \rightarrow 2 M$ o fluxo determinado pelo campo $Y$ dado pela proposição acima. Já que $X$ aponta para dentro nos pontos do bordo de $M$, fica definido em $M$ o semi-fluxo global $\psi$ do campo $X$ :

$$
\begin{aligned}
& \psi: \mathbb{R}_{+} \times M \rightarrow M \\
& \psi(t, x):=\phi(t, x)
\end{aligned}
$$

sendo $\phi$ o fluxo do campo $Y$ em $2 M$.

Se $M$ é uma $G$-variedade e $X$ é um $G$-campo de vetores em $M$, a propo- 
sição acima ainda se aplica, bastando para tanto substituirmos a partição da unidade usada na construção do campo $Y$ (cf. [Hirsch], pag. 151) por uma $G$-partição da unidade adequada. A existência de $G$-partições da unidade é garantida em [Bredon], pag. 133. E deste modo o comentário que segue a proposição acima continua aplicável pois, como já vimos, o fluxo de um $G$-campo é $G$-equivariante.

\subsection{G-Funções de Morse}

DEFINIÇÃO 1.6.1. Sejam $M$ uma variedade de dimensão $m$, com ou sem bordo e $f: M \rightarrow \mathbb{R}$ uma função de classe $\mathcal{C}^{\infty}$ e $p$ um ponto crítico de $f$, isto é, $d f_{p}=0$. Seja $(U, \varphi)$ uma carta em $p$. O hessiano de $f$ em $p$, em relação à $\operatorname{carta}(U, \varphi)$, é a matriz $(h)_{m \times m}$ dada por $h_{i j}=\frac{\partial^{2}\left(f \circ \varphi^{-1}\right)}{\partial x_{i} \partial x_{j}}(\varphi(p))$. Dizemos que o ponto crítico $p$ é não degenerado se o hessiano de $f$ em $p$ em relação à carta $(U, \varphi)$ é não singular, isto é, tem determinante diferente de zero. Pode-se mostrar que tal definição independe da escolha da carta $(U, \varphi)$.

DEFINIÇÃO 1.6.2. Uma função de Morse numa variedade $M$ é uma função $f: M \rightarrow \mathbb{R}$ cujos pontos críticos, se existirem, são não degenerados. Se a variedade $M$ possuir bordo a função $f: M \rightarrow \mathbb{R}$ será de Morse se todos os seus pontos críticos forem não degenerados e estiverem no interior de $M$.

PROPOSIÇÃO 1.6.3. Os pontos críticos não degenerados de uma função $f: M \rightarrow \mathbb{R}$ são isolados (cf. [Milnor], pag. 8). Portanto se $M$ é variedade compacta e $f$ é uma função de Morse, existe apenas um número finito de tais pontos críticos.

DEFINIÇÃO 1.6.4. Uma G-função de Morse em uma G-variedade $M$ é uma função de Morse $f: M \rightarrow \mathbb{R}$ que é invariante. 
O fato de que as funções de Morse são densas no conjunto de todas as funções de $M$ em $\mathbb{R}$ é um resultado clássico (cf. [Hirsch]). Abaixo enunciamos a versão equivariante deste fato. (cf. [Wasserman])

PROPOSIÇÃO 1.6.5. Seja $M$ uma G-variedade de dimensão finita. Então o conjunto das G-funções de Morse em $M$ é denso no conjunto de todas as funções invariantes de M. Em particular existem G-funcôes de Morse. 


\section{Capítulo 2}

\section{Realização de Conjuntos de Pontos Fixos}

Sejam $G$ um grupo finito e $M$ uma variedade $C^{\infty}$ de dimensão $n$, compacta e conexa, com ou sem bordo. Suponha que a ação de $G$ em $M$ é $C^{\infty}$. Mostraremos que se um subconjunto $P \subseteq \operatorname{int} M$, não vazio, $G$-invariante e compacto tem intersecção não vazia com cada componente conexa de $M_{H}$, para cada $H \leq G \operatorname{com} M_{H} \neq \emptyset$, então $P$ pode ser realizado como conjunto de pontos fixos de uma $G$-deformação. Chamaremos cada componente de $M_{H}$, com $H \leq G$, de uma componente de pontos fixos de isotropia $H$.

\subsection{Lema da Absorção}

Sejam $G$ um grupo finito e $M$ uma $G$-variedade. O lema abaixo garante que dado um subconjunto $P \subseteq \operatorname{int} M$, não vazio, finito e $G$-invariante, então para cada subconjunto finito $Q \subseteq \operatorname{int} M$ satisfazendo a condição (**) abaixo, existe sistema de cartas locais centradas nos pontos de $P$ cuja reunião das imagens em $M$ cobrem $Q$ e, além disso, são "bem comportadas" em relação à ação do grupo $G$. 
Lema da Absorção Seja $P \subset$ intM um subconjunto finito, $G$-invariante e não vazio. Seja $Q \subset$ intM um subconjunto finito tal que para cada $x \in Q$ a seguinte condição está satisfeita:

\section{(**) $C(x)$ tem intersecção não vazia $\operatorname{com} P$,}

sendo $C(x)$ a componente conexa de $M_{G_{x}}$ contendo $x$.

Então existe um sistema de cartas locais $\Psi:=\left\{\psi_{p}: D^{m} \rightarrow M \mid p \in P\right\}$ satisfazendo:

(1) $\psi_{p}(0)=p$

(2) se $p \neq p^{\prime}$, então $\psi_{p}\left(D^{m}\right) \cap \psi_{p^{\prime}}\left(D^{m}\right)=\emptyset$;

(3) se $p^{\prime}=g p$, com $g \in G$, então $\psi_{p^{\prime}}\left(D^{m}\right)=g \psi_{p}\left(D^{m}\right)$ e $\psi_{p^{\prime}}^{-1} \circ g \circ \psi_{p}: D^{m} \rightarrow D^{m}$ é a restrição de uma aplicação linear ortogonal;

(4) $Q \subset \bigcup_{p \in P} \psi_{p}\left(D^{m}\right)$.

Inicialmente observamos que a hipótese $Q \subseteq \operatorname{int} M$ não pode ser removida, como mostram os dois exemplos abaixo.

Exemplo 1: Considere o anel $A:=\left\{x \in \mathbb{R}^{2} \mid 1 \leq x \leq 2\right\}$ com a estrutura de variedade com bordo induzida de $\mathbb{R}^{2}$, os pontos $a=(-2,0), b=(-1,0)$, $c=(1,0), d=(2,0)$ e o grupo $\mathbb{Z}_{2}=\{1,-1\}$ agindo em $A$ por reflexão no eixo $x$, isto é:

$$
\forall(x, y) \in A, 1(x, y):=(x, y) \text { e }-1(x, y):=(x,-y)
$$

Sejam $p:=$ um ponto do interior do segmento $[a, b], P=\{p\}$ e $Q:=\{a, b\}$. Vemos que $P$ é $\mathbb{Z}_{2}$-invariante e de $A_{\mathbb{Z}_{2}}=[a, b] \cup[c, d]$, temos $C(b)=C(a)=[a, b]$. É claro que $P \cap C(a) \neq \emptyset$ e $P \cap C(b) \neq \emptyset$, no entanto não há nenhuma carta (centrada em $p$ ) contendo $p$ que contenha $a$ e $b$. 


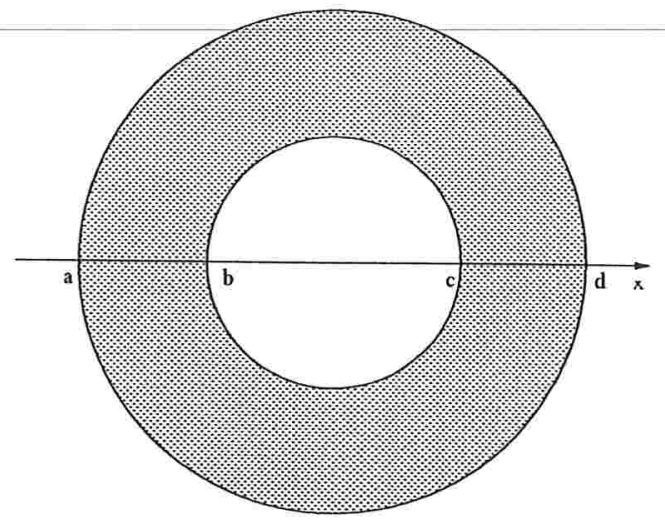

Exemplo 2: Mesmo no caso de ação trivial não podemos prescindir da hipótese $Q \subseteq \operatorname{int} M$. Basta considerar a variedade do exemplo acima, um grupo $G$ agindo trivialmente em $A$ e os mesmos conjuntos $P$ e $Q$ (nesse caso $C(a)=C(b)=A)$.

\section{Prova}

Faremos a prova por indução finita sobre a cardinalidade de $Q$.

Se $Q=\emptyset$, é resultado bem conhecido da geometria diferencial que podemos equipar $M$ com uma métrica riemanniana ( $G$-invariante) de tal modo que $\partial M$ fique totalmente geodésico, isto é, as geodésicas que passam pelos pontos do bordo tem imagens contidas no bordo. Seja $T_{p} M$ o espaço tangente de $M$ em $p$. A partir da aplicação exponencial exp: $T_{p} M \rightarrow M$ restrita a uma pequena bola em torno de $p$ podemos construir o sistema $\Psi$ desejado.

Suponhamos que $\Psi$ é sistema de cartas locais satisfazendo o lema para um conjunto $Q$ satisfazendo a condição (**) acima e tomemos $x \in \operatorname{int} M$. Mostraremos que $\Psi$ pode ser alterado de modo a satisfazer o lema com $Q \cup\{x\}$ no lugar de $Q$. Podemos assumir que $x \notin P \cup G Q$, pois caso contrário $\Psi$ 
não precisaria ser alterado: se $x \in P$, então $x \in \cup_{p \in P} \psi_{p}\left(D^{m}\right)$; se $x \in G Q$, então $\exists q \in Q$ tal que $x=g q$, logo existe $p \in P$ com $q \in \psi_{p}\left(D^{m}\right)$ e portanto $x=g q \in g \psi_{p}\left(D^{m}\right)=\psi_{g p}\left(D^{m}\right)=\psi_{p^{\prime}}\left(D^{m}\right) \operatorname{com} p^{\prime} \in P$.

Sejam $H:=G_{x}, S:=$ a componente conexa de $\left(M_{H} \cap \operatorname{int} M\right)$ contendo $x$ e $V:=\{z \in S \mid$ o lema está verificado com $Q$ substituido por $Q \cup\{z\}\}$. Vamos mostrar que $V=S$ via um argumento de conexidade. Observamos, inicialmente, que $V \neq \emptyset$, pois, por hipótese, $C(x) \cap P \neq \emptyset$ e $P \subset \operatorname{int} M$ e portanto $S \cap P \neq \emptyset$.

$V$ é aberto de $S$ : se $z \in V$, por hipótese $\exists \psi_{p} \in \Psi$ tal que $z \in \psi_{p}\left(D^{m}\right) \cap S$. Já que $\psi_{p}\left(D^{m}\right) \cap S$ é aberto de $S$ e para todo $w \in \psi_{p}\left(D^{m}\right) \cap S$, o lema está verificado para $Q \cup\{w\}$, vemos que $\psi_{p}\left(D^{m}\right) \cap S \subseteq V$.

Resta mostrar que $S \backslash V$ é também aberto de $S$ : dado $z \in S \backslash V$, então de modo análogo ao que foi feito para $Q=\emptyset$ podemos achar uma carta local $\phi: D^{m} \rightarrow M \backslash(P \cup G Q)$ tal que:

(1') $\phi(0)=z$;

(2') se $g \notin H$, então $\phi\left(D^{m}\right) \cap g \phi\left(D^{m}\right)=\emptyset$;

(3') se $g \in H$, então $\phi\left(D^{m}\right)=g \phi\left(D^{m}\right)$ e $\phi^{-1} \circ g \circ \phi: D^{m} \rightarrow D^{m}$ é a restrição de uma transformação linear ortogonal $A_{g}: \mathbb{R}^{m} \rightarrow \mathbb{R}^{m}$.

A ação de $H$ em $\mathbb{R}^{m}$ dada por $g v:=A_{g}(v), \forall g \in G, \forall v \in \mathbb{R}^{m}$ é a ação de $H$ em $\mathbb{R}^{m}$ induzida por $\phi$.

Seja $U:=S \cap \phi\left(D^{m}\right)$. Então: $\phi^{-1}(U)=D^{\ell}$, sendo $D^{\ell}$ o disco aberto unitário do subespaço vetorial $E^{\ell}:=\left(\mathbb{R}^{m}\right)^{H}$. De fato: se $v \in \phi^{-1}(U)$, então existe $w \in S \cap \phi\left(D^{m}\right)$ tal que $v=\phi^{-1}(w)$. Logo $\|v\|<1$ e para $g \in H, g(v)=A_{g}(v)=\phi^{-1} \circ g \circ \phi(v)=\phi^{-1}(g w)=v$, pois $G_{w}=H$. Portanto $v \in D^{\ell}$. Por outro lado, se $v \in D^{\ell}$, então existe $u \in \phi\left(D^{m}\right)$ tal que 
$\phi(v)=u$ e para todo $g \in H$ vale $g u=g \phi(v) \in g \phi\left(D^{m}\right)=\phi\left(D^{m}\right)$. Logo $\phi^{-1}(g u)=\phi^{-1}(g \phi(v))=A_{g}(v)=v=\phi^{-1}(u)$, e segue da bijeção de $\phi$ que $g u=u$. Concluimos, portanto, que $H \subseteq G_{u}$. Seja $g \in G_{u}$. Se $g \notin H$, da definição de $\phi$ temos $g \phi\left(D^{m}\right) \cap \phi\left(D^{m}\right)=\emptyset$ e portanto $g u \neq u$. Portanto $G_{u}=H$ e $u \in S \cap \phi\left(D^{m}\right)=U$, o que conclui a prova de que $\phi^{-1}(U)=D^{\ell}$.

Afirmamos que $U \subseteq S \backslash V$. Suponhamos, por absurdo, que a afirmação não é verdadeira. Sejam, $y \in U \cap V$ e $\theta$ um difeomorfismo $H$-equivariante de $D^{m}$ em $D^{m}$ que leva $\phi^{-1}(y)$ em $0=\phi^{-1}(z)$ e deixa pontos próximos de $\partial D^{m}$ fixados. (cf.[Bredon],pag. 314)

Consideremos a aplicação

$$
k:=\phi \circ \theta \circ \phi^{-1}: \phi\left(D^{m}\right) \rightarrow \phi\left(D^{m}\right)
$$

Claramente a aplicação $k$ é um difeomorfismo e deixa pontos próximos ao bordo de $\phi\left(D^{m}\right)$ fixados. Para verificarmos que $k$ é $H$-equivariante tomamos $\phi(v) \in \phi\left(D^{m}\right)$ e $g \in H$ obtendo:

$$
\begin{aligned}
& k(g \phi(v))=\phi \circ \theta \circ \phi^{-1}(g \phi(v)) \\
= & \phi \circ \theta \circ \underbrace{\phi^{-1} \circ g \circ \phi}_{A_{g}}(v)=(\phi \circ \theta)\left(A_{g}(v)\right) \\
= & \left(\phi \circ A_{g}\right)(\theta(v))=\left(\phi \circ \phi^{-1} \circ g \circ \phi \circ \theta\right)(v)=(g \circ \phi \circ \theta)(v) \\
= & \left(g \circ \phi \circ \theta \circ \phi^{-1} \circ \phi\right)(v)=\left(g \circ \phi \circ \theta \circ \phi^{-1}\right)(\phi(v)) \\
= & g\left(\phi \circ \theta \circ \phi^{-1}\right)(\phi(v))=g k(\phi(v))
\end{aligned}
$$

Podemos estender o difeomorfismo $k$ a um $G$-difeomorfismo $\bar{k}$ de $M$ em $M$ dado por :

$$
\bar{k}(x)= \begin{cases}g k g^{-1} & \text { se } x \in g \phi\left(D^{m}\right) \text { para algum } g \in G \\ x & \text { se } x \notin \bigcup_{g \in G} g \phi\left(D^{m}\right)\end{cases}
$$


Inicialmente verificamos que a aplicação $\bar{k}$ está bem definida:

Se $x \in g_{1} \phi\left(D^{m}\right) \cap g_{2} \phi\left(D^{m}\right)$, então existem $a$ e $b$ em $\phi\left(D^{m}\right)$ tais que $x=$ $g_{1} a=g_{2} b . \quad$ Logo $g_{1}^{-1} g_{2} b=a$ e por $\left(2^{\prime}\right) g_{1}^{-1} g_{2} \in H$. Então $g_{1} k\left(g_{1}^{-1} x\right)=$ $g_{1} k(a)=g_{1} k\left(g_{1}^{-1} g_{2} b\right)=g_{1}\left(g_{1}^{-1} g_{2}\right) k(b)=g_{2} k(b)=g_{2} k\left(g_{2}^{-1} x\right)$. O que mostra a afirmação anterior. Da definição de $\bar{k}$ vemos que pontos próximos ao bordo de $g \phi\left(D^{m}\right)$ são fixados, qualquer que seja $g \in G$. Além disso $\bar{k}$ é um difeomorfismo e leva $y$ em $z$ pois de $y \in \phi\left(D^{m}\right)=1 \phi\left(D^{m}\right)$ temos $\bar{k}(y)=1 k 1^{-1}(y)=k(y)=z$. Para mostrar que $\bar{k}$ é $G$-equivariante sejam $g_{1} \in G$ e $x \in M$. Se $x \notin \bigcup_{g \in G} g \phi\left(D^{m}\right)$, então $g_{1} x \notin \bigcup_{g \in G} \phi\left(D^{m}\right)$, pois se $g_{1} x \in g \phi\left(D^{m}\right)$ para algum $g \in G$, então $x \in g_{1}^{-1} g \phi\left(D^{m}\right)$ o que é contra a localização de $x$. Nesse caso $\bar{k}\left(g_{1} x\right)=g_{1} x=g_{1} \bar{k}(x)$. Se, porém, $x=g a$ para algum $g \in G$ e $a \in \phi\left(D^{m}\right)$, teremos $\bar{k}\left(g_{1} x\right)=g_{1} g k\left(\left(g_{1} g\right)^{-1} g_{1} x\right)=$ $g_{1} g k\left(g^{-1} g_{1}^{-1} g_{1} g a\right)=g_{1} g k(a)=g_{1} g k\left(g^{-1} x\right)=g_{1} \bar{k}(x)$.

Consideremos o sistema de cartas locais $\Psi^{\prime}:=\left\{\bar{k} \circ \psi_{p} \mid \psi_{p} \in \Psi\right\}$. Afirmamos que $\Psi^{\prime}$ satisfaz todas as condições do lema. De fato:

(1) Já que $\phi\left(D^{m}\right) \cap P=\emptyset$ e $P$ é $G$-invariante, segue que $P \cap g \phi\left(D^{m}\right)=\emptyset$, $\forall g \in G$. Logo $\bar{k}(p)=p, \forall p \in P$ e $\bar{k} \circ \phi_{p}(0)=\bar{k}(p)=p$.

(2) Para $p \neq p^{\prime}, \psi_{p}\left(D^{m}\right) \cap \psi_{p^{\prime}}\left(D^{m}\right)=\emptyset$.

Logo $\bar{k} \circ \psi_{p}\left(D^{m}\right) \cap \bar{k} \circ \psi_{p^{\prime}}\left(D^{m}\right)=\emptyset$, pois $\bar{k}$ é bijeção.

(3) Se $p^{\prime}=g p, g \in G$, então $g \circ \bar{k} \circ \psi_{p}\left(D^{m}\right)=\bar{k} \circ g \circ \psi_{p}\left(D^{m}\right)=\bar{k} \circ \psi_{p^{\prime}}\left(D^{m}\right) \mathrm{e}$ $\left(\bar{k} \circ \psi_{p^{\prime}}\right)^{-1} \circ g \circ\left(\bar{k} \circ \psi_{p}\right)=\psi_{p^{\prime}}^{-1} \circ(\bar{k})^{-1} \circ g \circ \bar{k} \circ \psi_{p}=\psi_{p^{\prime}}^{-1} \circ(\bar{k})^{-1} \circ \bar{k} \circ g \circ \psi_{p}=$ $\psi_{p^{\prime}}^{-1} \circ g \circ \psi_{p}: D^{m} \rightarrow D^{m}$ é a restrição de uma transformação linear ortogonal de $\mathbb{R}^{m}$ em $\mathbb{R}^{m}$.

(4) A carta $\phi$ foi tomada satisfazendo $\phi\left(D^{m}\right) \subseteq M \backslash(P \cup G Q)$. 
Logo $Q \cap g \phi\left(D^{m}\right)=\emptyset$ para todo $g \in G$. Dado $q \in Q$, seja $\psi_{p} \in \Psi$ tal que $q \in \psi_{p}\left(D^{m}\right)$. Da definição de $\bar{k}$ temos $\bar{k}(q)=q$ e portanto $q \in$ $\bar{k} \circ \psi_{p}\left(D^{m}\right)$. Já que $\bar{k}(y)=z$ e $y \in V$, existe $\psi_{p} \in \Psi$ tal que $y \in \psi_{p}\left(D^{m}\right)$ e portanto $z \in \bar{k} \circ \psi_{p}\left(D^{m}\right)$. Concluimos que $Q \cup z \subseteq \bigcup_{p \in P} \bar{k} \circ \psi_{p}\left(D^{m}\right)$.

Da definição de $V$ temos que $z \in V$. Absurdo, pois tomamos $z \in S \backslash V$. Logo $U \subseteq S \backslash V$ como afirmado .

Da definição de $U$, vemos que $U$ é aberto de $S$. Então $S \backslash V$ é aberto de $S$ e lembrando que $S$ é conexo obtemos $S=V$. O que conclui a demonstração.

\subsection{Retração Equivariante em $D^{m}$}

Lema da Retração Seja $D^{m}$ a bola unitária em $\mathbb{R}^{m}$ ou em $\mathbb{R}_{+}^{m}$. Suponha que $W$ é um campo de vetores $\mathcal{C}^{\infty}$ sobre $D^{m}$ que se anula em $\bar{D}_{r}^{m}:=\{x \in$ $\left.D^{m}:\|x\| \leq r\right\}$, sendo $0<r<1$. Seja $\theta: \mathbb{R} \rightarrow \mathbb{R}$ função de classe $C^{\infty}$ satisfazendo:

(i) $\theta^{\prime}(t)>0$, para todo $t \in \mathbb{R}$;

(ii)

$$
\theta(t)= \begin{cases}t-r & \text { se } t \leq(1+2 r) / 3 \\ t & \text { se } t \geq(2+r) / 3\end{cases}
$$

(iii) $\theta^{-1}$ é $C^{\infty}$.

Seja $k: D^{m} \rightarrow D^{m}$ a aplicação

$$
k(x)= \begin{cases}\frac{\theta(\|x\|) x}{\|x\|} & \text { se } x \in D^{m} \backslash \bar{D}_{r}^{m} \\ 0 & \text { se } x \in \bar{D}_{r}^{m}\end{cases}
$$


que colapsa o disco $\bar{D}_{r}^{m}$ na origem. O campo de vetores $W$ é alterado, via $k$, em $k_{*} W$ dado por:

$$
k_{*} W(x)= \begin{cases}\left.D k\right|_{k^{-1}(x)} W\left(k^{-1}(x)\right) & \text { se } x \neq 0 \\ 0 & \text { se } x=0\end{cases}
$$

Esse novo campo de vetores é $C^{\infty}$ em $D^{m}$ e coincide com o campo $W$ em pontos próximos de $\partial D^{m}$. Mais ainda: se $H$ é grupo finito agindo ortogonalmente em $D^{m}$ e o campo original $W$ é $H$-equivariante, então o campo $k_{*} W$ também é $H$-equivariante.

Prova Inicialmente observamos que existe uma função $\theta$ como requerida acima. Vamos começar mostrando que a aplicação $k$ estabelece bijeção entre os anéis

$A:=D^{m} \backslash \bar{D}_{r}^{m}=\left\{x \in D^{m} \mid r<\|x\|<1\right\}$ e $B:=D^{m} \backslash\{0\}$. De fato: da definição de $k$ temos, para $x \in A$,

$$
\|k(x)\|=\left\|\frac{\theta(\|x\|) x}{\|x\|}\right\|=|\theta(\|x\|)|=\theta(\|x\|)
$$

Sejam $x_{1}, x_{2} \in A \operatorname{com} x_{1} \neq x_{2}$. Se $\left\|x_{1}\right\| \neq\left\|x_{2}\right\|$, então $\theta\left(\left\|x_{1}\right\|\right) \neq \theta\left(\left\|x_{2}\right\|\right)$. O que mostra que $k\left(x_{1}\right) \neq k\left(x_{2}\right)$. Se $\left\|x_{1}\right\|=\left\|x_{2}\right\|$, então $k\left(x_{1}\right)=k\left(x_{2}\right)$ acarreta

$$
\frac{\theta\left(\left\|x_{1}\right\|\right) x_{1}}{\left\|x_{1}\right\|}=\frac{\theta\left(\left\|x_{1}\right\|\right) x_{2}}{\left\|x_{1}\right\|}
$$

o que só é possível se $x_{1}=x_{2}$. Portanto $k$ é injetora.

Para mostrar que $k$ é sobrejetora notamos que:

(i) Se $\frac{2+r}{3} \leq\|x\|<1$, então $k(x)=x$

(ii) Se $\frac{1+2 r}{3}<\|x\|<\frac{2+r}{3}$, então da definição de $\theta$ temos $(1-r) / 3<\|k(x)\|=\theta(\|x\|)<(2+r) / 3$. 
Dado $y \in D^{m} \backslash\{0\},(1-r) / 3<\|y\|<(2+r) / 3$, tomamos $x=\frac{\theta^{-1}(\|y\|) y}{\|y\|}$.

Novamente, da definição de $\theta$ vemos que $(1+2 r) / 3<\theta^{-1}(\|y\|)<$ $(2+r) / 3$. Portanto, observando que $\|x\|=\theta^{-1}(\|y\|)$, temos:

$$
k(x)=\frac{\theta(\|x\|) x}{\|x\|}=\frac{\theta\left(\theta^{-1}(\|y\|)\right)}{\theta^{-1}(\|y\|)} \frac{\theta^{-1}(\|y\|) y}{\|y\|}=y
$$

(iii) Se $r<\|x\| \leq(1+2 r) / 3$, então, da definição de $k$, temos:

$$
0<\theta(\|x\|)=\|x\|-r \leq(1-r) / 3
$$

Dado $y \in D^{m} \backslash\{0\}, 0<\|y\| \leq(1-r) / 3$, tomando $x=\frac{(r+\|y\|) y}{\|y\|}$ temos

$0<\|x\|=r+\|y\| \leq r+(1-r) / 3=(1+2 r) / 3 \mathrm{e}$ $k(x)=\frac{\theta(\|x\|) x}{\|x\|}=\frac{(\|x\|-r) x}{\|x\|}=\frac{(r+\|y\|-r)(r+\|y\|) y}{(r+\|y\|)\|y\|}=y$.

O que mostra que a aplicação $k$ é bijeção entre os anéis $A$ e $B$ acima definidos. Mais ainda: da argumentação anterior vemos que $k$ estabelece bijeções entre os seguintes pares de anéis:

$$
\begin{gathered}
A_{1}:=\left\{x \in D^{m} \mid r<\|x\| \leq(1+2 r) / 3\right\} \text { e } B_{1}:=\left\{x \in D^{m} \mid 0<\|x\| \leq(1-r) / 3\right\} \\
k(x)=\frac{(\|x\|-r) x}{\|x\|} \\
k^{-1}(x)=\frac{(\|x\|+r) x}{\|x\|} \\
A_{2}:=\left\{x \in D^{m} \mid(1+2 r) / 3<\|x\|<(2+r) / 3\right\}
\end{gathered}
$$

e

$$
B_{2}:=\left\{x \in D^{m} \mid(1-r) / 3<\|x\|<(2+r) / 3\right\}
$$




$$
\begin{gathered}
k(x)=\frac{\theta(\|x\|) x}{\|x\|} \\
k^{-1}(x)=\frac{\theta^{-1}(\|x\|) x}{\|x\|} \\
A_{3}:=\left\{x \in D^{m} \mid(2+r) / 3 \leq x<1\right\} \text { e } A_{3} \\
\left.k\right|_{A_{3}}=i d_{A_{3}}
\end{gathered}
$$

Observamos que as aplicações $k$ e $k^{-1}$ são de classe $C^{\infty}$ nos anéis $D^{m} \backslash \bar{D}_{r}^{m}$ e $D^{m} \backslash\{0\}$, respectivamente.

Para mostrar que $k_{*} W$ é campo de vetores de classe $C^{\infty}$ em $D^{m}$ basta mostrar que $k_{*} W$ é $C^{\infty}$ em $x=0$. Do exposto acima sabemos que $k^{-1} \mathrm{em}$ $\bar{D}_{(1-r) / 3}^{m} \backslash\{0\}$ é dada explicitamente por

$$
k^{-1}(x)=\frac{(r+\|x\|) x}{\|x\|}
$$

Afirmação: Se $u(x)$ é uma derivada parcial de ordem $n$ de uma componente de $k^{-1}$, então existe uma constante $c \geq 0$ tal que

$$
|u(x)| \leq c\|x\|^{-n} \text { para } 0<\|x\|<(1-r) / 3
$$

De fato: se $a_{i}$ é a $i$-ésima componente da aplicação $k^{-1}$, então da expressão de $k^{-1}(x)=\frac{r+\|x\|}{\|x\|}\left(x_{1}, \cdots, x_{m}\right)$, para $0<\|x\|<(1-r) / 3$, temos $a_{i}(x)=$ $\frac{r+\|x\|}{\|x\|} x_{i}=x_{i}+r x_{i}\|x\|^{-1}$ e observando que

$$
\frac{\partial\|x\|}{\partial x_{k}}=\frac{\partial}{\partial x_{k}}\left(\sum_{i=1}^{m} x_{i}^{2}\right)^{1 / 2}=\frac{1}{2}\left(\sum_{i=1}^{m} x_{i}^{2}\right)^{-1 / 2} \cdot 2 x_{k}=x_{k}\|x\|^{-1}
$$

temos, para $n=1$ : 


$$
\begin{aligned}
& \frac{\partial a_{i}}{\partial x_{i}}(x)=1+r\|x\|^{-1}-r x_{i}^{2}\|x\|^{-3} \\
& \frac{\partial a_{i}}{\partial x_{k}}(x)=-r x_{k} x_{i}\|x\|^{-3}, \text { se } i \neq k .
\end{aligned}
$$

Já que $\left|x_{i}\right| \leq\|x\|, \forall i=1, \cdots, m$ e $0<\|x\|<(1-r) / 3<1$ temos:

$$
\begin{gathered}
\left|\frac{\partial a_{i}}{\partial x_{i}}(x)\right| \leq\left(\|x\|+r+r x_{i}^{2}\|x\|^{-2}\right)\|x\|^{-1} \leq\left(\|x\|+r+r\|x\|^{2}\|x\|^{-2}\right)\|x\|^{-1}= \\
=(\|x\|+2 r)\|x\|^{-1} \leq(1+2 r)\|x\|^{-1} \\
\left|\frac{\partial a_{i}}{\partial x_{k}}(x)\right| \leq r\left|x_{k}\left\|x_{i} \mid\right\| x\left\|^{-3} \leq r\right\| x\left\|^{2}\right\| x\left\|^{-3}=r\right\| x \|^{-1}\right.
\end{gathered}
$$

Tomando $c=1+2 r$ no primeiro caso e $c=r$ no segundo, teremos a limitação desejada para uma derivada de ordem 1 de $a_{i}(x)$.

Vamos mostrar que para todo inteiro $n \geq 2$ existem funções polinomiais $p_{b}: \mathbb{R}^{m} \rightarrow \mathbb{R}, b=n, \cdots, 2 n+1$ satisfazendo (a) e (b) abaixo:

(a) Se $p_{b}$ é não nulo, $g r\left(p_{b}\right)=b-n$

(b) $\frac{\partial^{n} a_{i}}{\partial x_{j_{1}}^{s_{1}} \cdots \partial x_{j_{\ell}}^{s_{\ell}}}(x)=\sum_{b=n}^{2 n+1} p_{b}(x)\|x\|^{-b}$

Para $n=2$ temos:

- $\frac{\partial^{2} a_{i}}{\partial x_{i}^{2}}=-3 r x_{i}\|x\|^{-3}+3 r x_{i}^{3}\|x\|^{-5}$ e tomando $p_{2}(x)=0, p_{3}(x)=-3 r x_{i}$ $p_{4}(x)=0$ e $p_{5}(x)=3 r x_{i}^{3}$ vemos que (a) e (b) estão verificados.

- $\frac{\partial^{2} a_{i}}{\partial x_{i} \partial x_{k}}(x)=\frac{\partial^{2} a_{i}}{\partial x_{k} \partial x_{i}}(x)=-r x_{k}\|x\|^{-3}+3 r x_{k} x_{i}^{2}\|x\|^{-5}$ e tomando $p_{2}(x)=$ $0, p_{3}(x)=-r x_{k}, p_{4}(x)=0, p_{5}(x)=3 r x_{k} x_{i}^{2}$ teremos os ítens (a) e (b) verificados.

- $\frac{\partial^{2} a_{i}}{\partial x_{t} \partial x_{k}}(x)=\frac{\partial^{2} a_{i}}{\partial x_{k} \partial x_{t}}(x)=3 r x_{i} x_{k} x_{t}\|x\|^{-5}$ e tomando $p_{2}(x)=0, p_{3}(x)=$ $0, p_{4}(x)=0$ e $p_{5}(x)=3 r x_{i} x_{k} x_{t}$ teremos os ítens $(\mathrm{a})$ e $(\mathrm{b})$ verificados. 
- $\frac{\partial^{2} a_{i}}{\partial x_{k}^{2}}=-r x_{i}\|x\|^{-3}+3 r x_{i} x_{k}^{2}\|x\|^{-5}$ e tomando $p_{2}(x)=0, p_{3}(x)=-r x_{i}$, $p_{4}(x)=0$ e $p_{5}(x)=3 r x_{i} x_{k}^{2}$ vemos que (a) e (b) estão verificados.

Suponhamos, por hipótese, que (a) e (b) estão verificados para algum inteiro $n \geq 2$. Então

$$
\begin{gathered}
\frac{\partial}{\partial x_{t}}\left(\frac{\partial^{n} a_{i}}{\partial x_{j_{1}}^{s_{1}} \cdots \partial x_{j_{\ell}}^{s_{\ell}}}\right)(x)=\frac{\partial}{\partial x_{t}}\left(\sum_{b=n}^{2 n+1} p_{b}(x)\|x\|^{-b}\right)= \\
=\sum_{b=n}^{2 n+1} \frac{\partial p_{b}}{\partial x_{t}}(x)\|x\|^{-b}+\sum_{b=n}^{2 n+1} p_{b}(x) \frac{\partial\|x\|^{-b}}{\partial x_{t}} \\
=\sum_{b=n}^{2 n+1} \frac{\partial p_{b}}{\partial x_{t}}(x)\|x\|^{-b}+\sum_{b=n}^{2 n+1}-b x_{t} p_{b}(x)\|x\|^{-(b+2)}
\end{gathered}
$$

pois $\frac{\partial\|x\|^{-b}}{\partial x_{t}}=-b\|x\|^{-(b+1)}\|x\|^{-1} x_{t}=-b x_{t}\|x\|^{-(b+2)}$.

Levando em conta que $\frac{\partial p_{n}}{\partial x_{t}}$ é nulo, pois, por hipótese, $p_{n}$ é nulo ou tem grau zero e reagrupando os termos da soma acima teremos:

$$
\begin{gathered}
\frac{\partial}{\partial x_{t}}\left(\frac{\partial^{n} a_{i}}{\partial x_{j_{1}}^{s_{1}} \cdots \partial x_{j_{\ell}}^{s_{\ell}}}\right)(x)=\frac{\partial}{\partial x_{t}}\left(\sum_{b=n}^{2 n+1} p_{b}(x)\|x\|^{-b}\right)= \\
=\frac{\partial p_{n+1}}{\partial x_{t}}(x)\|x\|^{-(n+1)}+\left(\frac{\partial p_{n+2}}{\partial x_{t}}(x)-n x_{t} p_{n}(x)\right)\|x\|^{-(n+2)}+\cdots \\
\cdots+\left(\frac{\partial p_{2 n+1}}{\partial x_{t}}(x)-(2 n-1) x_{t} p_{2 n-1}(x)\right)\|x\|^{-(2 n+1)}+ \\
-2 n x_{t} p_{2 n}(x)\|x\|^{-(2 n+2)}-(2 n+1) x_{t} p_{2 n+1}(x)\|x\|^{-(2 n+3)}
\end{gathered}
$$

Sejam

$$
q_{n+1}:=\frac{\partial p_{n+1}}{\partial x_{t}}
$$




$$
\begin{aligned}
& q_{n+2+k}:=\frac{\partial p_{n+2+k}}{\partial x_{t}}-(n+k) x_{t} p_{n+k}, \text { se } 0 \leq k \leq n-1 \\
& q_{2 n+2}:=-2 n x_{t} p_{2 n} \\
& q_{2 n_{3}}:=-(2 n+1) x_{t} p_{2 n+1}
\end{aligned}
$$

Então podemos escrever:

$$
\frac{\partial}{\partial x_{t}}\left(\frac{\partial^{n} a_{i}}{\partial x_{j_{1}}^{s_{1}} \cdots \partial x_{j_{\ell}}^{s_{\ell}}}\right)(x)=\sum_{b=n+1}^{2(n+1)+1} q_{b}(x)\|x\|^{-b}
$$

Portanto os polinômios $q_{b}$, para $b=n+1, \cdots, 2 n+3$, acima definidos verificam o item (b). Para mostrar que também verificam (a) observamos que:

- $q_{n+1}$ é nulo ou tem grau zero, pois, por hipótese, $p_{n+1}$ é nulo ou tem grau 1.

- Para $q_{n+2+k}, 0 \leq k \leq n-1$ temos: $p_{n+2+k}$ é nulo ou tem grau $k+2$, $p_{n+k}$ é nulo ou tem grau $k$ e além disso, cada fator ocorrendo nesses polinômios tem o mesmo grau do respectivo polinômio. Portanto $\frac{\partial p_{n+2+k}}{\partial x_{t}}$ é nulo ou tem grau $k+1$ e cada um de seus fatores, nesse caso, também tem grau $k+1$. Se $p_{n+k}$ não é nulo, então $(n+k) x_{t} p_{n+k}$ e cada um de seus fatores tem grau $k+1$. Logo $q_{n+2+k}$ é nulo ou tem grau $k+1$ com cada um de seus fatores com grau $k+1$.

- Para $q_{2 n+2}$ notamos que se $p_{2 n}$ não é nulo, então $g r\left(p_{2 n}\right)=n$ e portanto $g r\left(q_{2 n+2}\right)=n+1$ e, além disso, cada fator de $q_{2 n+2}$ terá grau $n+1$, pois, cada fator de $p_{2 n}$ tem, por hipótese, grau $n$.

- De modo análogo ao caso anterior podemos mostrar que $q_{2 n+1}$ é nulo ou tem grau $n+2$, o mesmo ocorrendo com cada um de seus fatores. 
Portanto, para todo $n \geq 2$, vale:

$$
\left|\frac{\partial^{n} a_{i}}{\partial x_{j_{1}}^{s_{1}} \cdots \partial x_{j_{\ell}}^{s_{\ell}}}(x)\right| \leq \sum_{b=n}^{2 n+1}\left|p_{b}(x)\right|\|x\|^{-b}
$$

Se $x=\left(x_{1}, \cdots, x_{m}\right)$, então $\left|x_{i}\right| \leq\|x\|, \forall i=1,2 \cdots, m$ e portanto $\left|p_{b}(x)\right| \leq c_{b}\|x\|^{b-n}$, sendo $c_{b}$ constante $\geq 0$. Então

$$
\left|\frac{\partial^{n} a_{i}}{\partial x_{j_{1}}^{s_{1}} \cdots \partial x_{j_{\ell}}^{s_{\ell}}}(x)\right| \leq \sum_{b=n}^{2 n+1} c_{b}\|x\|^{b-n}\|x\|^{-b}=\sum_{b=n}^{2 n+1} c_{b}\|x\|^{-n}=c\|x\|^{-n}
$$

sendo $c=\sum_{b=1}^{2 n+1} c_{b}$. Lembrando que o caso $n=1$ já foi verificado, vemos que a afirmação está, portanto, justificada, i.e., se $u(x)=\frac{\partial^{n} a_{i}}{\partial x_{j_{1}}^{s_{1}} \cdots \partial x_{j_{\ell}}^{s_{\ell}}}(x)$, então existe constante $c$ tal que $|u(x)| \leq c\|x\|^{-n}$ para todo $x$ com $0<\|x\|<(1-r) / 3$.

Sejam $w_{i}(x)$ a i-ésima componente do campo $W(x)$ e $q(x)$ uma derivada de ordem $n$ de $w_{i}(x)$. Já que $W$ é de classe $C^{\infty}$ em $D^{m}$ e nulo em $\bar{D}_{r}^{m}$, segue que para qualquer $z \in D^{m}$ com $\|z\|=r$, qualquer derivada de $w_{i}(x)$ é nula em $z$. Seja $y_{0} \in D^{m} \backslash \bar{D}_{r}^{m}$. Dado $y \in \bar{D}_{\left\|y_{0}\right\|}^{m}$ indicaremos com $z_{y}$ o ponto de intersecção do segmento $[0, y] \operatorname{com} \partial\left(\bar{D}_{r}^{m}\right)$.

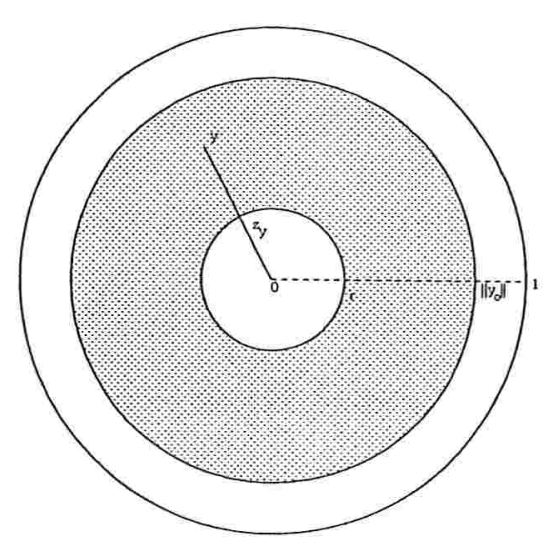


Dado inteiro $n \geq 1$, existe $M \geq 0$ tal que $\left\|q^{(n+1)}(y)\right\| \leq M$, para todo $y$ em $\in \bar{D}_{\left\|y_{0}\right\|}^{m}$, pois $W$ é $C^{\infty}$. Usando a fórmula de Taylor com resto de Lagrange ([Lima1], pag. 58), com $h=y-z_{y}$, temos:

$$
q(y)=q\left(z_{y}\right)+q^{\prime}\left(z_{y}\right) h+\frac{1}{2 !} q^{\prime \prime}\left(z_{y}\right) h^{(2)}+\cdots \frac{1}{n !} q^{(n)}\left(z_{y}\right) h^{(n)}+r(h)
$$

sendo $\|r(h)\| \leq \frac{M}{(n+1) !}\|h\|^{n+1}$ e indicando por $h^{(k)}$ o vetor $(\underbrace{h, \cdots, h)}_{k \text {-vezes }}$ e por $q^{(k)}\left(z_{y}\right) h^{(k)}$ o resultado da aplicação $k$-linear $q^{(k)}\left(z_{y}\right)$ no vetor $h^{(k)}$.

Já que $q(z)=q^{\prime}(z)=q^{\prime \prime}(z)=\cdots=q^{(n)}(z)=0$ vemos que $q(y)=r(h)$ e portanto $|q(y)| \leq \frac{M}{(n+1) !}\|h\|^{n+1}=c(\|y\|-r)^{n+1} \operatorname{com} c=M /(n+1)$ !

A aplicação $\left.k\right|_{D^{m} \backslash \bar{D}_{r}^{m}}$ tem uma extensão $\hat{k}: D^{m} \backslash\{0\} \rightarrow D^{m}$ que é dada por $\hat{k}(y)=\frac{\theta(\|y\|) y}{\|y\|}$ de classe $C^{\infty}$. Conseqüentemente $\left.D k\right|_{y}$ e todas as suas derivadas parciais são limitadas em $D^{m} \backslash \bar{D}_{r}^{m}$.

Finalmente, podemos mostrar que $k_{*} W$ é de classe $C^{\infty}$ na origem. Seja $x \in D^{m}$ tal que $0<\|x\|<(1-r) / 3$. Da prova que $k$ é bijeção entre $D^{m} \backslash\{0\}$ e $D^{m} \backslash \bar{D}_{r}^{m}$ temos:

(1) $y=k^{-1}(x) \in A_{1}:=\left\{x \in D^{m} \mid r<\|x\| \leq(1+2 r) / 3\right\}$

(2) $y=k^{-1}(x)=\frac{(r+\|x\|) x}{\|x\|}$ e $k(y)=\frac{(\|y\|-r) y}{\|y\|}$

(3) $\|x\| \rightarrow 0 \Rightarrow\left\|k^{-1}(x)\right\| \rightarrow r$ e portanto $x \rightarrow 0 \Rightarrow W\left(k^{-1}(x)\right) \rightarrow 0$.

(4) $\left.D k\right|_{k^{-1}(x)} W\left(k^{-1}(x)\right)=\left(\frac{\partial k_{i}}{\partial x_{j}} k^{-1}(x)\right)_{m \times m} \times\left(\begin{array}{c}w_{1}\left(k^{-1}(x)\right) \\ \vdots \\ w_{m}\left(k^{-1}(x)\right)\end{array}\right)$ 
Sendo $k_{i}$ e $w_{i}$ as componentes $i$-ésimas de $k$ e $W$, respectivamente.

Observamos que do último item temos

$$
\left.D k\right|_{k^{-1}(x)} W\left(k^{-1}(x)\right)=\left(\sum_{j=1}^{m} w_{j}\left(k^{-1}(x)\right) \frac{\partial k_{i}}{\partial x_{j}}\left(k^{-1}(x)\right)\right)_{i=1, \cdots, m} \in \mathbb{R}^{m}
$$

Seja $d_{i}(x)$ a $i$-ésima componente de $\left.D k\right|_{k^{-1}(x)} W\left(k^{-1}(x)\right)$, isto é, $d_{i}(x)=\sum_{j=1}^{m} \frac{\partial k_{i}}{\partial x_{j}}\left(k^{-1}(x)\right) w_{j}\left(k^{-1}(x)\right)$.

Temos, portanto:

(i) Da limitação de $D k \mid y$ e de todas as suas derivadas parciais em $D^{m} \backslash \bar{D}_{r}^{m}$, de $r<\|y\|<(1+2 r) / 3$ e de (3) anterior, vemos que $x \rightarrow 0$ acarreta $d_{i}(x) \rightarrow 0$.

(ii)

$$
\frac{\partial d_{i}}{\partial x_{p}}(x)=\sum_{j=1}^{m}\left[w_{j}\left(k^{-1}(x)\right) \frac{\partial^{2} k_{i}}{\partial x_{p} \partial x_{j}}\left(k^{-1}(x)\right)+\frac{\partial k_{i}}{\partial x_{j}}\left(k^{-1}(x)\right) \frac{\partial w_{j}}{\partial x_{p}}\left(k^{-1}(x)\right)\right]
$$

Observando que $\frac{\partial w_{j}}{\partial x_{p}}\left(k^{-1}(x)\right)=\sum_{\ell=1}^{m} \frac{\partial w_{j}}{\partial x_{\ell}}\left(k^{-1}(x)\right) \frac{\partial k_{\ell}^{-1}}{\partial x_{p}}(x)$ e fazendo $k^{-1}(x)=y$ podemos reescrever $\frac{\partial d_{i}}{\partial x_{p}}(x)$ como

$$
\frac{\partial d_{i}}{\partial x_{p}}(x)=\sum_{j=1}^{m}\left[w_{j}(y) \frac{\partial^{2} k_{i}}{\partial x_{p} \partial x_{j}}(y)+\frac{\partial k_{i}}{\partial x_{j}}(y) \sum_{\ell=1}^{m} \frac{\partial w_{j}}{\partial x_{\ell}}(y) \frac{\partial k_{\ell}^{-1}}{\partial x_{p}}(x)\right]
$$

Logo

$$
\left|\frac{\partial d_{i}}{\partial x_{p}}(x)\right| \leq \sum_{j=1}^{m}\left|\frac{\partial^{2} k_{i}}{\partial x_{p} \partial x_{j}}(y)\right|\left|w_{j}(y)\right|+\sum_{j, \ell=1}^{m}\left|\frac{\partial k_{i}}{\partial x_{j}}(y)\right|\left|\frac{\partial w_{j}}{\partial x_{\ell}}(y)\right|\left|\frac{\partial k_{\ell}^{-1}}{\partial x_{p}}(x)\right|
$$


Usando as limitações (⿳) e (⿳) temos:

$$
\left|\frac{\partial k_{\ell}^{-1}}{\partial x_{p}}(x)\right| \leq c_{1}\|x\|^{-1} \text { e }\left|\frac{\partial w_{j}}{\partial x_{\ell}}(y)\right| \leq c_{2}(\|y\|-r)^{2}
$$

Portanto $\left|\frac{\partial w_{j}}{\partial x_{\ell}}(y)\right|\left|\frac{\partial k_{\ell}^{-1}}{\partial x_{p}}(x)\right| \leq c\|x\|^{-1}(\|y\|-r)^{2}$, com $c=c_{1} c_{2}$. Usando (2) acima temos:

$c\|x\|^{-1}(\|y\|-r)^{2}=c\|x\|^{-1}(r+\|x\|-r)^{2}=c\|x\|^{-1}\|x\|^{2}=c\|x\|$.

Já que $w_{j}(y)=w_{j}\left(k^{-1}(x)\right) \rightarrow 0$ quando $x \rightarrow 0,\left.D k\right|_{y}$ e todas as suas derivadas parciais são limitadas em $D^{m} \backslash \bar{D}_{r}^{m}$ temos:

$$
\left|\frac{\partial d_{i}}{\partial x_{p}}(x)\right| \leq\left(\sum_{j=1}^{m}\left|\frac{\partial^{2} k_{i}}{\partial x_{p} \partial x_{j}}(y)\right|\left|w_{j}(y)\right|+c \sum_{j, \ell=1}^{m}\left|\frac{\partial k_{i}}{\partial x_{j}} y\right|\|x\|\right) \rightarrow 0, \text { se } x \rightarrow 0
$$

Podemos ver que qualquer derivada parcial de orden $n$ de $d_{i}(x)$ consistirá de uma soma de produtos semelhantes aos que ocorrem em $\frac{\partial d_{i}}{\partial x_{p}}(x)$. Portanto, usando argumento análogo, podemos mostar que qualquer derivada parcial de ordem $n$ de $d_{i}(x)$ terá limite nulo para $x \rightarrow 0$. Deste modo concluimos que $k_{*} W(x)$ é $C^{\infty}$ na origem e de $k(x)=x$ para $x \in A_{3}$ vemos que $k_{*} W$ coincide com $W$ em pontos próximos de $\partial D^{m}$.

Resta mostrar que se $H$ é grupo agindo ortogonalmente em $D^{m}$ e $W$ é $H$-equivariante, então $k_{*} W$ também é $H$-equivariante.

Como $\|g x\|=\|x\|, \forall x \in D^{m}, \forall g \in H$, temos:

$k_{*} W(g 0)=k_{*} W(0)=0=g k_{*} W(0)$ e se $x \in D^{m} \backslash\{0\}$, então $g x \neq 0 \mathrm{e}$ $k_{*} W(g x)=\left.D k\right|_{k^{-1}(g x)} W\left(k^{-1}(g x)\right)$.

Há duas possibilidades para $x \in D^{m} \backslash\{0\}: x \in D^{m} \backslash \bar{D}_{r}^{m}$ e $x \in \bar{D}_{r}^{m}$.

- Se $x \in D^{m} \backslash \bar{D}_{r}^{m}$, então $k(g x)=\frac{\theta(\|g x\|) g x}{\|g x\|}=\frac{\theta(\|x\|) g x}{\|x\|}=g(k x)$, pois $H$ age linearmente.

- Se $x \in \bar{D}_{r}^{m}$, então $g x \in \bar{D}_{r}^{m}$ e portanto $g k(x)=g 0=0=k(g x)$. 


\subsection{Realização de Conjuntos como Conjuntos de Pontos Fixos} de uma $G$-deformação

Logo $k$ é $H$-equivariante. Como observado no início da demonstração, $k$ estabelece difeomorfismo $C^{\infty}$ entre $D^{m} \backslash \bar{D}_{r}^{m}$ e $D^{m} \backslash\{0\}$, portanto $k^{-1}$ de $D^{m} \backslash\{0\}$ em $D^{m} \backslash \bar{D}_{r}^{m}$ é $H$-equivariante.

Voltando à expressão de $k_{*} W(g x)$ e usando a linearidade da aplicação $x \mapsto g x$ temos:

$$
\begin{gathered}
k_{*} W(g x)=\left.D k\right|_{k^{-1}(g x)} W\left(k^{-1}(g x)\right)=\left.D k\right|_{g k^{-1}(x)} W\left(g k^{-1}(x)\right)= \\
=\left.D k\right|_{g k^{-1}(x)} g W\left(k^{-1}(x)\right)=\left.\left.D k\right|_{g k^{-1}(x)} D g\right|_{k^{-1}(x)} W\left(k^{-1}(x)\right)= \\
=\left.D k g\right|_{k^{-1}(x)}\left(W\left(k^{-1}(x)\right)=\left.D g k\right|_{k^{-1}(x)} W\left(k^{-1}(x)\right)=\right. \\
=\left.\left.D g\right|_{k^{-1}} D k\right|_{k^{-1}(x)} W\left(k^{-1}(x)\right)=\left.g D k\right|_{k^{-1}(x)} W\left(k^{-1}(x)\right)=g k_{*} W(x)
\end{gathered}
$$

$\mathrm{O}$ que mostra a equivariância de $k_{*} W$

\subsection{Realização de Conjuntos como Conjuntos de Pontos Fixos de uma $G$-deformação}

Teorema Sejam $M$ uma $G$-variedade com ou sem bordo e $P \subset$ int $M$ um subconjunto compacto, não vazio e $G$-invariante. Suponhamos que, para todo subgrupo $H$ de $G$, o subconjunto $P$ tenha intersecção não vazia com todas as componentes de pontos fixos de isotropia $H$, se $M_{H} \neq \emptyset$. Então existe um $G$-campo de vetores $V$ em $M$ tal que $Z_{V}=P$ e cujas órbitas (do campo de vetores) não são fechadas.

Prova: A existência de uma função de Morse $G$-equivariante $\varphi: M \rightarrow \mathbb{R}$ está garantida pela Proposição 1.6.5. Seja $X$ o campo de vetores em $M$ dado pelo gradiente de $\varphi$. Já que as singularidades de $X$ são os pontos críticos da função $\varphi$, que são não degenerados e estão no interior de $M$ (cf. Definição 1.6.2), segue da Proposição 1.6.3 que $X$ tem apenas um número finito de 


\subsection{Realização de Conjuntos como Conjuntos de Pontos Fixos} de uma $G$-deformação

singularidades e, além disso, o campo $X$ é $G$-equivariante e não possui órbitas não singulares fechadas, conforme as Proposições 1.5.18 e 1.5.19.

Seja $Q$ o conjunto das singularidades de $X$. Como já vimos $Q \subset \operatorname{int} M$ e é finito. Seja $P^{\prime} \subseteq P$, finito, tal que $Q$ satisfaz a condição (**) do lema da absorção em relação a $P^{\prime}$. Seja $\Psi=\left\{\psi_{p}: D^{m} \rightarrow M \mid p \in P^{\prime}\right\}$ o sistema de cartas locais dado pelo lema da absorção, com $P^{\prime}$ no lugar de $P$. Sejam $r$ número real, $0<r<1$, tal que $Q \subset \bigcup_{p \in P^{\prime}} \psi_{p}\left(D_{r}^{m}\right)$ e $b: \mathbb{R} \rightarrow \mathbb{R}$ uma função $\mathcal{C}^{\infty}$ satisfazendo $b(t)=0$ para $t \leq r, b(t)>0$ para $t>r$, e $b(t)=1$ para $t \geq(1+r) / 2$. Usando $b$ definimos a função $\bar{b}: M \rightarrow \mathbb{R}$ por

$$
\bar{b}(x)= \begin{cases}1 & \text { se } x \notin \bigcup_{p \in P^{\prime}} \psi_{p}\left(D^{m}\right) \\ b\left(\left\|\psi_{p}^{-1}(x)\right\|\right) & \text { se } x \in \psi_{p}\left(D^{m}\right)\end{cases}
$$

A função $\bar{b}$ está bem definida e é de classe $\mathcal{C}^{\infty}$. Consideremos o campo de vetores $W$ em $M$ dado por $W(x)=\bar{b}(x) X(x)$. Tal campo $W$ é $G^{-}$ equivariante. De fato: se $x \notin \bigcup_{p \in P^{\prime}} \psi_{p}\left(D^{m}\right)$, então $g x \notin \bigcup_{p \in P^{\prime}} \psi_{p}\left(D^{m}\right)$ e já que os campos $X$ e $W$ coincidem no complementar de $\bigcup_{p \in P^{\prime}} \psi_{p}\left(D^{m}\right)$, segue que $W(g x)=X(g x)=\left.D g\right|_{x} X(x)=\left.D g\right|_{x} W(x)$. Se, por outro lado, $x \in \psi_{p}\left(D^{m}\right)$ para algum $p \in P^{\prime}$, lembrando que $\psi_{g p}\left(D^{m}\right)=g \psi_{p}\left(D^{m}\right)$ e que $\psi_{g p}^{-1} \circ g \circ \psi_{p}$ é uma aplicação linear ortogonal de $D^{m}$ em $D^{m}$, obtemos $W(g x)=$ $\bar{b}(g x) X(g x)=b\left(\left\|\psi_{g p}^{-1}(g x)\right\|\right) X(g x)=b\left(\left\|\left(\psi_{g p}^{-1} \circ g \circ \psi_{p}\right)\left(\psi_{p}^{-1}(x)\right)\right\|\right) X(g x)=$ $b\left(\left\|\psi_{p}^{-1}(x)\right\|\right) X(g x)=\left.b\left(\left\|\psi_{p}^{-1}(x)\right\|\right) D g\right|_{x}(X(x))=\left.D g\right|_{x}\left(b\left(\left\|\psi_{p}^{-1}(x)\right\|\right) X(x)\right)=$ $\left.D g\right|_{x}(W(x))$.

As órbitas de $W$ em $\bigcup_{p \in P^{\prime}} \psi\left(\bar{D}_{r}^{m}\right)$ são todas constantes pois $W$ é nulo em tal subconjunto de $M$. Todas as outras órbitas de $W$ são reparametrizações de partes de órbitas de $X$, portanto não são fechadas.

Vamos usar o lema da retração para colapsar cada "disco" $\psi_{p}\left(\bar{D}_{r}^{m}\right)$, com 


\subsection{Realização de Conjuntos como Conjuntos de Pontos Fixos} de uma $G$-deformação

$p \in P^{\prime}$, em um único ponto. Para tanto definimos a função $\bar{k}: M \rightarrow M$ do seguinte modo:

$$
\bar{k}(x)= \begin{cases}x & \text { se } x \notin \bigcup_{p \in P^{\prime}} \psi_{p}\left(D^{m}\right) \\ \psi_{p} k \psi_{p}^{-1}(x) & \text { se } x \in \psi_{p}\left(D^{m}\right) \text { para algum } p \in P^{\prime}\end{cases}
$$

sendo $k: D^{m} \rightarrow D^{m}$ como no lema da retração. Portanto a aplicação $\bar{k}$ está bem definida e, além disso, é $G$-equivariante. De fato:

- Se $x \notin \bigcup_{p \in P^{\prime}} \psi_{p}\left(D^{m}\right)$, então $g x \notin \bigcup_{p \in P^{\prime}} \psi_{p}\left(D^{m}\right)$, para qualquer $g \in G$, e portanto $\bar{k}(g x)=g x=g \bar{k}(x)$.

- Se $\exists p \in P^{\prime}$ tal que $x \in \psi_{p}\left(D^{m}\right)$, então, $g x \in \psi_{g p}\left(D^{m}\right)=g \psi_{p}\left(D^{m}\right)$ e portanto $\bar{k}(g x)=\psi_{g p} k \psi_{g p}^{-1}(g x)=\psi_{g p} \circ k \circ \psi_{g p}^{-1} \circ g \circ \psi_{p}\left(\psi_{p}^{-1}(x)\right)$. Mas a aplicação $k$ comuta com toda transformação ortogonal de $D^{m}$ ( final da demonstração do lema da retração) e como a aplicação $\psi_{g p}^{-1} \circ g \circ \psi_{p}$ é ortogonal temos: $\psi_{g p} \circ k \circ \psi_{g p}^{-1} \circ g \circ \psi_{p} \circ k\left(\psi_{p}^{-1}(x)\right)=g \psi_{p} k \psi_{p}^{-1}(x)=g \bar{k}(x)$. Logo $\bar{k}(g x)=g \bar{k}(x)$.

Consideremos o novo campo de vetores $Y$ em $M$ dado por $Y=\bar{k}_{*} W$, isto é, $Y(x)=\left.D \bar{k}\right|_{\bar{k}^{-1}(x)} W\left(\bar{k}^{-1}(x)\right)$. Vemos que $Z_{Y}=P^{\prime}$, isto é, o conjunto de singularidades do campo $Y$ é $P^{\prime}$ e usando argumento análogo ao empregado no fim da demostração do lema da retração podemos mostrar que esse novo campo é $G$-equivariante.

Seja $a: M \rightarrow \mathbb{R}$ função $\mathcal{C}^{\infty}$ tal que $\left.a\right|_{P} \equiv 0$ e $a(x)>0$ para todo $x \in M \backslash P$. A partir da função a construimos a função $\bar{a}: M \rightarrow \mathbb{R}$ dada por $\bar{a}(x)=$ $\frac{1}{|G|} \sum_{g \in G} a(g x)$. A função $\bar{a}$ está bem definida e é $\mathcal{C}^{\infty}$. Quanto à equivariância temos: $\bar{a}(g x)=\frac{1}{|G|} \sum_{h \in G} a(h g x)=\frac{1}{|G|} \sum_{h \in G} a(h x)=g \frac{1}{|G|} \sum_{h \in G} a(h x)=$ $g \bar{a}(x)$. Finalmente, o campo de vetores $V$ em $M$ dado por $V(x)=\bar{a}(x) Y(x)$ é $\mathcal{C}^{\infty}, G$-equivariante e $Z_{V}=P$. O que conclui a prova do teorema. 


\subsection{Realização de Conjuntos como Conjuntos de Pontos Fixos}

de uma $G$-deformação

Corolário (Realização de conjuntos como conjunto de pontos fixos de uma $G$-deformação) Sejam $M$ uma $G$-variedade com ou sem bordo e $P \subset$ intM um subconjunto compacto e $G$-invariante com interseç̧ão não vazia com toda componente não vazia de pontos fixos de isotropia $H$, para todo subgrupo $H$ de $G$. Então $P$ é o conjunto de pontos fixos de uma G-deformação.

Prova Do teorema anterior decorre a existência de um $G$-campo de vetores $X$ em $M$ tal que $Z_{X}=P$ e todas as órbitas não singulares de $X$ não são fechadas; em particular $X$ não possui órbitas periódicas. Dos comentários após a Proposição 1.5.22, o campo $X$ admite um semi-fluxo $\left\{\phi_{t}\right\}_{t \geq 0}$. Pela Proposição 1.5.13 tal semi-fluxo é $G$-equivariante. Então $\phi_{0}=i d_{M}$ e dado $t>0$ temos $\phi_{t}(x)=x$ se $x \in P$ e $\phi_{t}(x) \neq x$ se $x \notin P$. Portanto, dado $t>0$, $P=\operatorname{Fix}\left(\phi_{t}\right)$, isto é, $P$ é realizado como conjunto de pontos fixos de uma $G$-deformação. 


\section{Capítulo 3}

\section{O Exemplo de D. Ferrario}

O objetivo inicial desta dissertação era a apresentação do artigo A note on equivariant vector fields de Boju Jiang ([Jiang]) que trata, em linhas gerais, da realização de subconjuntos compactos e $G$-invariantes de uma $G$ variedade compacta, conexa, com ou sem bordo, como conjuntos de pontos fixos de $G$-deformações. A única hipótese assumida sobre tais subconjutos, além da compacidade e $G$-invariância, é a intersecção não vazia com cada componente conexa de característica de Euler não nula de cada $M^{G_{x}}$. Sendo verdadeiro tal teorema de realização, obteríamos, como corolário, tomando como subconjunto a ser realizado o conjunto vazio, um resultado importante na teoria de pontos fixos que é obtido na literatura com hipóteses adicionais sobre, por exemplo, a dimensão, co-dimensão e conexidade dos subespaços $M^{H}$. O resultado seria o seguinte:

Seja $M$ uma $G$-variedade compacta e conexa, com ou sem bordo. Se a identidade de $M^{H}$ pode ser deformada a uma aplicação livre de pontos fixos, para cada $H \leq G$, então a identidade de $M$ pode ser $G$-deformada a uma aplicação livre de pontos fixos. 
Porém um exemplo apresentado por Ferrario em sua tese de doutorado (cf. [Ferrario]) mostra que o resultado acima não é verdadeiro e portanto o teorema de realização do artigo mencionado não está correto, pelo menos, para o caso do conjunto vazio. O exemplo apresentado em [Ferrario] consta de uma $G$-variedade em que todos os subespaços $M^{H}$ são conexos, têm característica de Euler nula e, portanto, todas as suas identidades podem ser deformadas a aplicações livres de pontos fixos, no entanto a identidade de $M$ não pode ser $G$-deformada a uma aplicação livre de pontos fixos. Mais ainda: o exemplo mostrou que outros resultados no artigo poderiam estar comprometidos pois neles está assumida uma premissa que não é verificada no exemplo mencionado, apesar deste satisfazer todas as hipóteses pedidas no artigo. Um de tais resultados é o Engulfing Lemma, apresentado na dissertação como lema da absorção após uma mudança de hipótese que permitiu sua demonstração na linha apresentada no artigo.

A argumentação dada em [Ferrario] de que a identidade da $G$-variedade, compacta e conexa, apresentada como exemplo não pode ser $G$-deformada a uma aplicação livre de pontos fixos, utiliza o conceito de indíce equivariante de ponto fixo, número de Nielsen equivariante, etc. Já que esta dissertação não seguiu tal linha, apresentaremos um critério dado por Komiya em [Komiya] com o qual analisaremos tal exemplo :

Seja $M$ uma $G$-variedade compacta e sem bordo, sendo $G$ um grupo de Lie compacto. $M$ admite um $G$-campo de vetores sem singularidades se, e somente se, a característica de Euler de cada componente conexa de $M_{G_{x}} / N\left(G_{x}\right)$ é nula, para todo $x \in M$.

Decorre do teorema acima e de um resultado bem conhecido de topolo- 
gia diferencial ([Lima2]) que, nestas condições, para $t>0$ suficientemente pequeno $\phi_{t}: M \rightarrow M$ não possui pontos fixos, sendo $\phi: R \times M \rightarrow M$ o fluxo do campo dado, que é $G$-equivariante pela Proposição 1.5.13.

Passemos ao exemplo.

Seja $G$ o grupo diedral de ordem $6, D_{3}$, nos geradores $r_{1}$ e $r_{2}$ :

$$
G:=\left\langle r_{1}, r_{2} \mid r_{1}^{2}=r_{2}^{2}=\left(r_{2} r_{2}\right)^{3}=1\right\rangle=\left\{1, r_{1}, r_{2}, r_{1} r_{2},\left(r_{1} r_{2}\right)^{2}, r_{2} r_{1} r_{2}\right\}
$$

Os subgrupos de $G$ são:

$$
\begin{aligned}
& H_{1}:=\left\langle r_{1}\right\rangle=\left\{1, r_{1}\right\} \\
& H_{2}:=\left\langle r_{2}\right\rangle=\left\{1, r_{2}\right\} \\
& H_{3}:=\left\langle r_{2} r_{1} r_{2}\right\rangle=\left\{1, r_{2} r_{1} r_{2}\right\} \\
& H_{4}:=\left\langle r_{1} r_{2}\right\rangle=\left\{1, r_{1} r_{2},\left(r_{1} r_{2}\right)^{2}\right\}
\end{aligned}
$$

Já que $H_{4}$ tem índice 2 em $G$ vemos que é normal. Além disso verificamos que $N\left(H_{i}\right)=H_{i}$ para $i=1,2,3$.

Consideremos o plano cartesiano $V:=\mathbb{R}^{2}$ e duas retas, designadas, também, por $r_{1}$ e $r_{2}$, que formam ângulo de $\pi / 3$. Consideremos, também, a reta $t$ obtida por reflexão de $r_{2}$ em $r_{1}$ (ou vice-versa). Façamos $G$ agir em $V$ por reflexão nas retas $r_{1}$ e $r_{2}$, usando $r_{i} \in G$ para designar a reflexão na reta $r_{i}, \operatorname{com} i=1,2$.

Para calcularmos as isotropias da ação de $G$ em $V$ observamos que:

$$
\begin{aligned}
& p \in V \backslash\left(r_{1} \cup r_{2} \cup t\right) \Rightarrow g p=p \Longleftrightarrow g=1 \\
& p \in r_{1} \Rightarrow r_{2} p \in t, r_{1} r_{2} p \in r_{2} \text { e } r_{2} r_{1} r_{2} p=r_{1} r_{2} p \in r_{2}
\end{aligned}
$$


$p \in r_{2} \Rightarrow r_{1} p \in t, r_{1} r_{2} p \in t$ e $r_{2} r_{1} r_{2} p \in t$

$p \in t \Rightarrow r_{1} p \in r_{2}, r_{2} p \in r_{1}, r_{1} r_{2} p \in r_{1}$ e $r_{2} r_{1} r_{2} p=p$

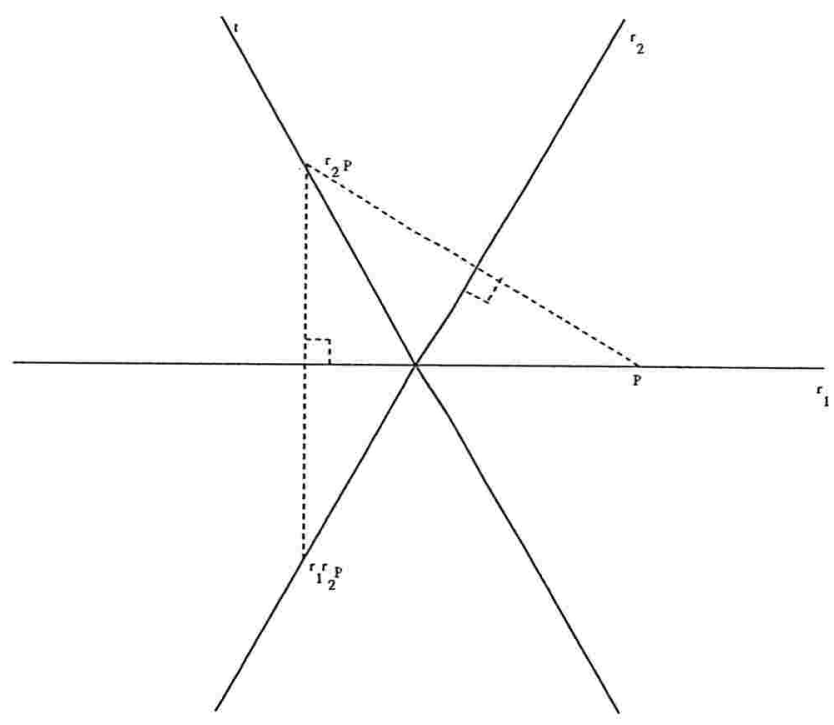

Fig. 4.1 A ação de $G=D_{3}$ no plano $V=\mathbb{R}^{2}$

Usando as observações acima obtemos:

$$
\begin{aligned}
& p \in r_{1}, p \neq(0,0) \Rightarrow G_{p}=H_{1} \\
& p \in r_{2}, p \neq(0,0) \Rightarrow G_{p}=H_{2} \\
& p \in t, p \neq(0,0) \Rightarrow G_{p}=H_{3} \\
& p \in V \backslash\left(r_{1} \cup r_{2} \cup t\right) \Rightarrow G_{p}=\{1\}
\end{aligned}
$$

Além disso: $V^{H_{1}}=r_{1}, V^{H_{2}}=r_{2}, V^{H_{3}}=t, V^{H_{4}}=V^{G}=\{(0,0)\}$.

Seja $W=V \oplus \mathbb{R}^{3}$ com a ação de $G$ dada por

$$
g(p, z):=(g p, z), \forall g \in G, \forall p \in V, \forall z \in \mathbb{R}^{3}
$$

É fácil verificar que: 


$$
\begin{aligned}
& W^{G}=(0,0) \times \mathbb{R}^{3}=W^{H_{4}} \approx \mathbb{R}^{3} \\
& W^{H_{1}}=r_{1} \times \mathbb{R}^{3} \approx \mathbb{R}^{4} \\
& W^{H_{2}}=r_{1} \times \mathbb{R}^{3} \approx \mathbb{R}^{4} \\
& W^{H_{3}}=t \times \mathbb{R}^{3} \approx \mathbb{R}^{4}
\end{aligned}
$$

Consideremos um toro $T^{2}$ e $D^{2}$ um disco cujo bordo é uma curva simples, $C$, em $T$. Podemos mergulhar $T^{2}$ em $W^{H_{1}}$ de tal modo que $T^{2} \cap W^{G}=C$.

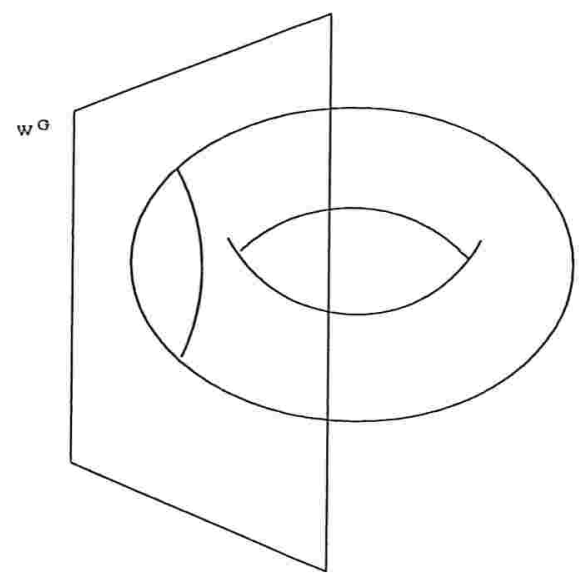

Fig. $4.2 \mathrm{O}$ toro $T^{2}$ mergulhado em $W^{H_{1}}$ e o plano $W^{G}$

Então $r_{1} T^{2}=T^{2}, r_{2} T^{2}$ é um toro em $W^{H_{3}}$ e $r_{1} r_{2} T$ é um toro em $W^{H_{2}}$. Já que $r_{2} r_{1} r_{2} T^{2}=r_{1} r_{2} T^{2}$ temos: $G T^{2}=T^{2} \cup r_{2} T^{2} \cup r_{1} r_{2} T^{2}$ e $T^{2} \cap r_{2} T^{2}=T^{2} \cap r_{1} r_{2} T^{2}=r_{2} T^{2} \cap r_{1} r_{2} T^{2}=C=T^{2} \cap W^{G}$. Abaixo está uma ilustração do que está ocorrendo, fazendo-se algumas adaptações em relação as dimensões envolvidas.

Considere $X$ uma $G$-vizinhança regular e $G T^{2}$ que é $G$-deformável a $G T^{2}$ (cf. [RS]); podemos escolher $X$ uma variedade diferenciável, compacta, com 


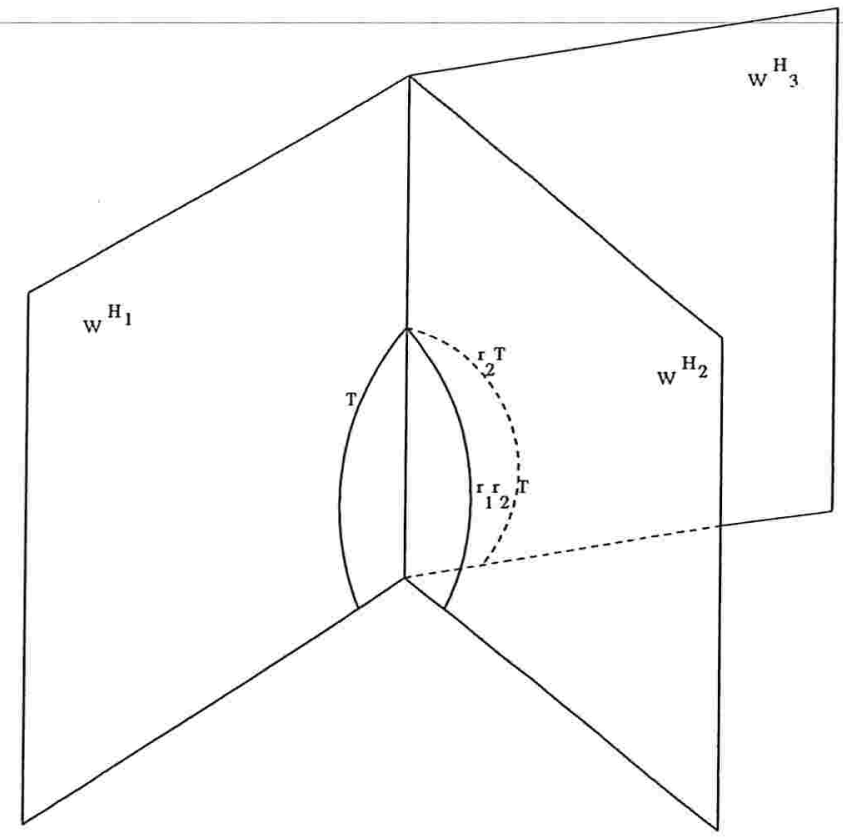

Fig. 4.3 os toros $T^{2}, r_{1} T^{2}$ e $r_{1} r_{2} T^{2}$

bordo e de dimensão 5. Para o cálculo das isotropias da ação de $G$ em $W$ observamos que $X^{H_{1}}$ é uma vizinhança regular de $T^{2}$ em $W^{H_{1}} \approx \mathbb{R}^{4}$, logo $X^{H_{1}}=T^{2} \times D^{2}$. Análogamente $X^{H_{2}}=r_{1} r_{2} T^{2} \times D^{2}$ e $X^{H_{3}}=r_{2} T^{2} \times D^{2}$, ou seja $X^{H_{1}} \approx X^{H_{2}} \approx X^{H_{3}}$. Além dissso $X^{G}=X^{H_{4}}=C \times D^{2}$, pela mesma razão.

Seja $Y=2 X$ a $G$-variedade compacta, conexa, sem bordo e de dimensão 5 obtida colando duas cópias de $X$ identificadas pelo bordo via a identidade. Vamos calcular $\chi\left(Y^{H}\right)$ para os grupos de isotropia $H \leq G$. Sabemos que $\chi(Y)=2 \chi(X)-\chi(\partial X)$. Já que $G T^{2}$ é um retrato por deformação de $X$ temos: $\chi(X)=\chi\left(G T^{2}\right)=0$. Como a dimensão de $X$ é 5 , usando dualidade de Lefschetz (cf. [Massey], pag. 227 e ex. 7.3) temos $\chi(\partial X)=0$. Portanto $\chi(Y)=0$. O subespaço $Y^{H_{1}}$ é a soma de duas cópias de $X^{H_{1}}$ com os bordos 
identificados pela identidade, isto é,

$$
Y^{H_{1}}=T^{2} \times D^{2} \bigcup_{T^{2} \times S^{1}} T^{2} \times D^{2}=T^{2} \times S^{2}
$$

sendo $S^{2}$ a esfera de dimensão 2. Logo $\chi\left(Y^{H_{1}}\right)=0 \mathrm{e}$, analogamente, $\chi\left(Y^{H_{2}}\right)=\chi\left(Y^{H_{3}}\right)=0$.

Já que $X^{G}=C \times D^{2}$ e $Y^{G}=C \times D^{2} \bigcup_{C \times S^{1}} C \times D^{2}=C \times S^{2}$, vemos que $\chi\left(Y^{G}\right)=\chi\left(Y^{H_{4}}\right)=0$. Além disso, $Y_{G}=Y^{G}=C \times S^{2}$ e $Y_{H_{i}}=Y^{H_{i}} \backslash Y^{G}, i=$ 1,2,3. Em particular

$$
Y_{H_{1}}=Y^{H_{1}} \backslash Y^{G}=\left(T^{2} \times S^{2}\right) \backslash\left(C \times S^{2}\right)=\left(T^{2} \backslash C\right) \times S^{2}
$$

Então $Y_{H_{1}}$ tem duas componentes conexas: $A_{1} \approx D^{2}$ e $A_{2}$ que tem o mesmo tipo de homotopia do buquê $S^{1} \vee S^{1}$. Já que $N\left(H_{1}\right)=H_{1}$, temos

$$
Y_{H_{1}} / N\left(H_{1}\right)=Y_{H_{1}} / H_{1}=\left(\left(T^{2} \backslash C\right) \times S^{2}\right) / H_{1}
$$

Mas a ação de $H_{1}$ em $Y^{H_{1}}$ é trivial. Logo

$$
Y_{H_{1}} / N\left(H_{1}\right) \approx A_{1} \times S^{2} \cup A_{2} \times S^{2} \approx D^{2} \times S^{2} \cup A_{2} \times S^{2}
$$

Mas $\chi\left(D^{2} \times S^{2}\right)=2$ e $\chi\left(A_{2} \times S^{2}\right)=-2$. Portando, pelo critério estabelecido em [Komiya] e citado no início deste capítulo, não existe um $G$-campo de vetores sobre $Y$ sem singularidades, o que contradiz o teorema 1(B) de [Jiang]. Além disso, David Ferrario demonstrou que a identidade de $Y$ não pode ser $G$-deformada a uma aplicação livre de pontos fixos, contrariando o corolário do teorema 2 de [Jiang]. 


\section{Bibliografia}

[BG] Borsari, Lucília Daruiz e Gonçalves, Daciberg Lima. G-deformation to fixed point free maps via obstruction theory. Preprint.

[Boothby] Boothby, Willian Munger. An introduction to differentiable manifolds and Riemannian geometry. Academic Press, Orlando; 1986.

[Bredon] Bredon, Glen E. Introduction to compact transformations groups, Academic Press, New York, 1972.

[Ferrario] D. Ferrario, Equivariant Deformations of Maps and Fixed Points, Tese de doutorado, 1999.

[FW] Fadell, E. e Wong, P. On deforming G-maps to be fixed point free, Pacific J. Math. 132, no. 2, 277-281.

[Halpern] Halpern, Benjamin Periodic points on tori, Pacific J. Math 83 (1979), no. 1, 117-133.

[Hirsch] Hirsch, Morris W. Differential Topology. Springer-Verlag, New York; 1976.

[IV] Izydorek, M e Vidal, A. A note on the converse of the Lefschetz theorem for G-maps, Ann. Polonici Math. LVII.2 (1983), 177-183.

[Jiang] Jiang, Boju. A note on equivariante vector fields, Acta Math. Scientia 11 (1991), no. 3, 274-282.

[Kawakubo] Kawakubo, Katsuo. The theory of transformations groups, Oxford; New York: Oxford University Press, 1991.

[Komiya] Komiya, Katsuhiro. A necessary and sufficient condition for the existence of non-singular $G$-vector fields on $G$-manifolds. Osaka J. Math. 13 (1976), 537-546. 
[Lima1] Lima, Elon Lages. Análise no espaço $\mathbb{R}^{n}$. Edgard Blücher, São Paulo; 1970.

[Lima2] Lima, Elon Lages. Introdução à topologia diferncial. IMPA, Rio de Janeiro, 1961.

[Massey] Massey, William S. Singular homology theory. Springer-Verlag, New York, 1980.

[Mil] Milnor, John Willard. Morse theory. Princeton University Press; 1969. [Palis] Palis Jr., Jacob e Melo, Welington. Introdução aos sistemas dinâmicos. IMPA, Rio de Janeiro; 1975.

[Plante] Plante, J. F. Diffeomorphisms without periodic points, Proc. AMS 88 (1983), no. 4, 716-718.

[RS] Rourke, C.P. e Sanderson, B. J. Introduction to Piecewise-linear topology. Springer-Verlag, Berlin, 1978.

[Schirmer] Schirmer, Helga. Fixed point sets of polyhedra. Pacific Journal of Mathematics, 52, No.1, 1974.

[tD] tom Dieck, Tammo. Transformation groups, Walter de Gruyter \& co., Berlin, 1987.

[Vidal] Vidal, A. On equivariante deformation of maps, Pub. Mat. 32 (1988), 115-121.

[Wang] Wang, Shichang e Xue, Tong. Fixed points sets of diffeomorphisms on compact differential manifolds. Adv. in Math. (Beijin) 12 (1983), 233-236.

[Wasserman] Wasserman, Arthur G. Equivariant Differential Topology. Topology 8 (1969) 127-150.

[Wilczyński] Wilczyński, Dariusz Fixed point free equivariant homotopy classes, Fund. Math. 123 (1984), 47-60. 